\title{
Utility of the Nudged Elastic Band Method in Identifying the Minimum Energy Path of an Elementary Organometallic Reaction Step
}

Kate E. McPherson, Libero J. Bartolotti, Andrew T. Morehead, Jr. and Andrew L. Sargent

Department of Chemistry, East Carolina University, Greenville, North Carolina

\section{SUPPORTING INFORMATION AVAILABLE}

- Experimental Methods: Computed energies, optimized molecular geometries, and harmonic frequencies were calculated with the numerical density functional package DMol3. ${ }^{3 \mathrm{~b}, 3 \mathrm{c}}$ The BOP (Becke-Tsuneda-Hirao) gradient-corrected functional ${ }^{3 \mathrm{~d}, 3 \mathrm{e}}$ was employed along with double numerical plus polarization basis sets, a 20 Bohr radial cutoff, a fine integration grid, and all-electron scalar relativistic corrections. ${ }^{3 \mathrm{f}}$ Convergence criteria of $10^{-9}$ hartrees for the SCF and $5 \times 10^{-4}$ hartrees/bohr for the gradient in geometry optimizations were utilized. Initial NEB calculations, such as the ones seen in Figure 1, are broken down into subsequent NEB calculations that focus on the pathway between two adjacent minima that are connected by a single transition state. While NEB can be run to convergence to obtain the transition state, in practice only a few hundred NEB cycles are run, the last one hundred or so with the climbing image option activated (Alfonso, D. R.; Jordan, K. D. J. Comput. Chem. 2003, 24, 990-996) to ensure that the highest of the movable images is as close to the transition state as possible. This image, along with additional data from the NEB calculation, is utilized in the Modified Dimer program (Henkelman, G.; Jónsson, J. J. Chem. Phys. 1999, 111, 7010-7022.; Heyden, A.; Bell, A.T.; Keil, F.J. J. Chem. Phys. 2005, $123,224101-14)$ to locate the transition state. Dimer is a local surface-walking algorithm that calculates only the lowest eigenvalue and eigenvector, rather than the full Hessian. The dimer vector, in our case, is defined by the two NEB images (the estimate of the TS and an 
adjacent image or point defining the tangent vector) and is rotated to locate the lowest curvature mode. The algorithm then steps in this direction uphill toward the saddle point.

- Table S1. Cartesian coordinates and DFT/BOP/DNP energies of optimized molecular structures from Figure 2, and structure 2d from Figure 1. 
Table S1. Cartesian coordinates and DFT/BOP/DNP energies of optimized molecular structures from Figure 2, and structure 2d from Figure 1. Units are in Angstroms, energies in Hartrees.

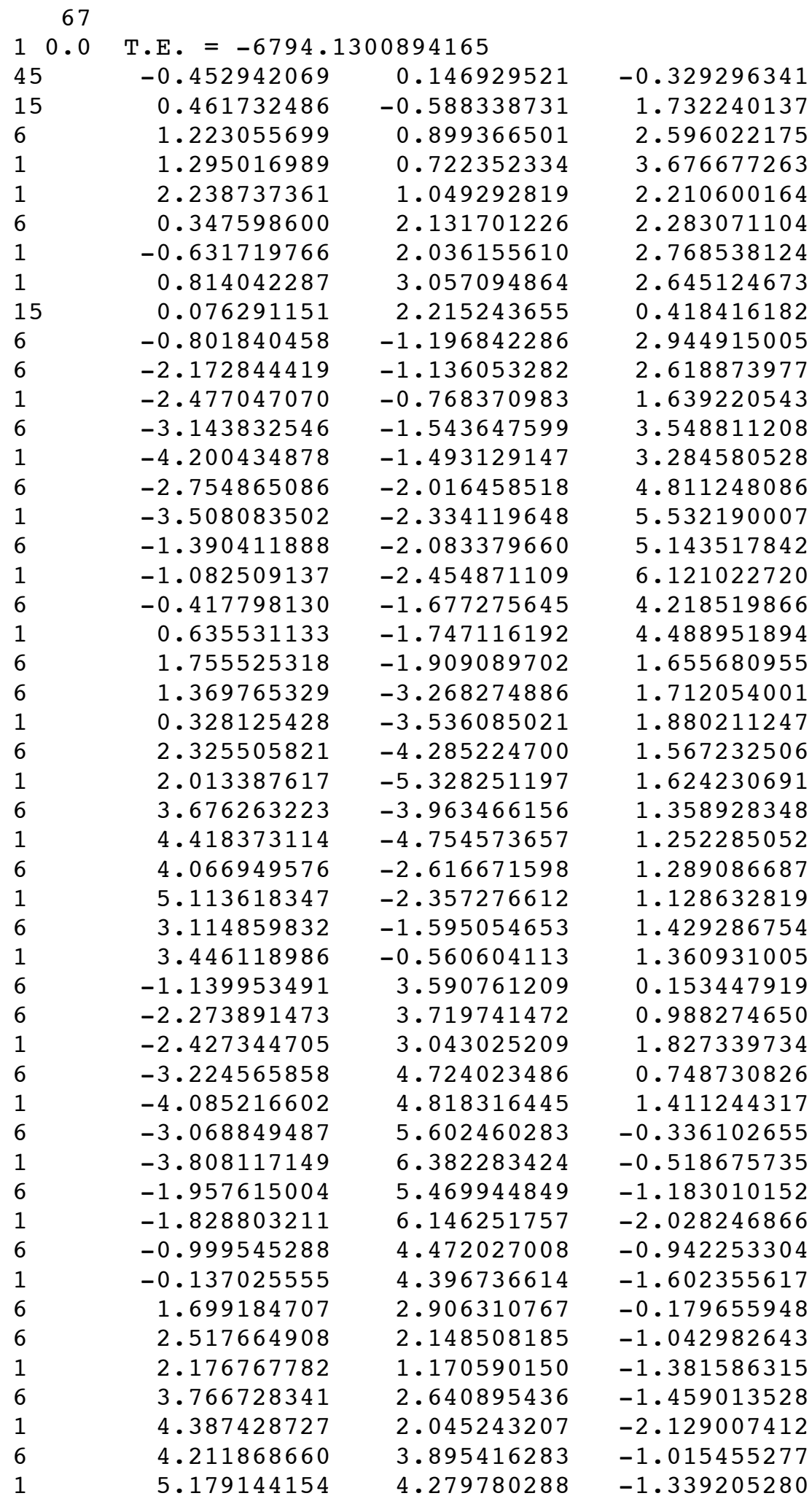




\begin{tabular}{|c|c|c|c|}
\hline 6 & 3.405376230 & 4.657214879 & -0.152084979 \\
\hline 1 & 3.745431879 & 5.632782269 & 0.195877420 \\
\hline 6 & 2.157889910 & 4.169194795 & 0.262900678 \\
\hline 1 & 1.543116849 & 4.781886327 & 0.921499227 \\
\hline 8 & -0.977840509 & -1.927289110 & -0.840583309 \\
\hline 6 & -1.231861157 & -2.467089751 & -1.931418066 \\
\hline 1 & -1.583864496 & -3.516184291 & -1.903738831 \\
\hline 6 & -1.128332784 & -1.829133376 & -3.290703705 \\
\hline 1 & -2.168347568 & -1.701382631 & -3.646317903 \\
\hline 1 & -0.704944407 & -2.586644670 & -3.970902490 \\
\hline 6 & -0.328972607 & -0.513778047 & -3.396703417 \\
\hline 1 & -0.377065337 & -0.185625833 & -4.449033480 \\
\hline 1 & 0.735312715 & -0.724987830 & -3.208124198 \\
\hline 6 & -0.768781470 & 0.661483251 & -2.532428716 \\
\hline 1 & -0.204979169 & 1.571960913 & -2.741556190 \\
\hline 6 & -1.977203410 & 0.792329529 & -1.845815754 \\
\hline 1 & -2.728490755 & 0.000992428 & -1.850098895 \\
\hline 1 & -2.351387723 & 1.776901643 & -1.576091322 \\
\hline \multicolumn{4}{|c|}{67} \\
\hline \multicolumn{4}{|c|}{$2 \mathrm{pi}+6.8 \quad$ T.E. $=-6794.1193145131$} \\
\hline 45 & -0.653106672 & 0.126890660 & -0.194215655 \\
\hline 15 & 0.392334573 & -0.575038873 & 1.802363988 \\
\hline 6 & 1.304683038 & 0.900856813 & 2.524570239 \\
\hline 1 & 1.485059962 & 0.757657229 & 3.597519932 \\
\hline 1 & 2.279872540 & 0.986607683 & 2.030391614 \\
\hline 6 & 0.464414387 & 2.168582203 & 2.264821200 \\
\hline 1 & -0.467168275 & 2.138071330 & 2.843382070 \\
\hline 1 & 1.009906518 & 3.075460873 & 2.555636491 \\
\hline 15 & 0.029262791 & 2.240319513 & 0.432086711 \\
\hline 6 & -0.831743308 & -1.032118239 & 3.115261680 \\
\hline 6 & -2.213494716 & -1.029083531 & 2.830060924 \\
\hline 1 & -2.559494717 & -0.808036912 & 1.821688702 \\
\hline 6 & -3.144911132 & -1.342136223 & 3.834251587 \\
\hline 1 & -4.209820046 & -1.341824378 & 3.600890911 \\
\hline 6 & -2.706230378 & -1.656124100 & 5.129316913 \\
\hline 1 & -3.429695286 & -1.897308052 & 5.907945288 \\
\hline 6 & -1.330885001 & -1.664996572 & 5.420353629 \\
\hline 1 & -0.984269066 & -1.921393054 & 6.421375396 \\
\hline 6 & -0.396745049 & -1.357977627 & 4.420801538 \\
\hline 1 & 0.665758239 & -1.386087866 & 4.661277110 \\
\hline 6 & 1.593515508 & -1.976720393 & 1.723073527 \\
\hline 6 & 1.126817431 & -3.301506495 & 1.884458843 \\
\hline 1 & 0.079931275 & -3.490127191 & 2.113920913 \\
\hline 6 & 2.010974101 & -4.383807205 & 1.758822784 \\
\hline 1 & 1.637818028 & -5.399224217 & 1.891750049 \\
\hline 6 & 3.366683706 & -4.162213730 & 1.468189716 \\
\hline 1 & 4.052512516 & -5.004402030 & 1.377754228 \\
\hline 6 & 3.836275571 & -2.849931614 & 1.296109787 \\
\hline 1 & 4.887633250 & -2.668506191 & 1.072528448 \\
\hline 6 & 2.956773184 & -1.762807439 & 1.415519633 \\
\hline 1 & 3.346558838 & -0.757556507 & 1.266177527 \\
\hline 6 & -1.214571711 & 3.601438091 & 0.258890562 \\
\hline 6 & -2.300949810 & 3.677761564 & 1.161656309 \\
\hline 1 & -2.384498029 & 2.981336385 & 1.994527816 \\
\hline 6 & -3.295543728 & 4.653295523 & 0.997750674 \\
\hline
\end{tabular}




\begin{tabular}{|c|c|c|c|}
\hline 1 & -4.120317346 & 4.705618763 & 1.708489663 \\
\hline 6 & -3.231447347 & 5.555114482 & -0.077084159 \\
\hline 1 & -4.004966949 & 6.312489940 & -0.202901347 \\
\hline 6 & -2.167031646 & 5.476382820 & -0.988295319 \\
\hline 1 & -2.110062911 & 6.171538887 & -1.826006378 \\
\hline 6 & -1.164550557 & 4.506496973 & -0.825262471 \\
\hline 1 & -0.342029594 & 4.469514621 & -1.537363230 \\
\hline 6 & 1.598756529 & 2.869971488 & -0.332932662 \\
\hline 6 & 2.337163263 & 2.045873757 & -1.206963948 \\
\hline 1 & 1.958890393 & 1.055597035 & -1.455047706 \\
\hline 6 & 3.556317655 & 2.488930407 & -1.746362725 \\
\hline 1 & 4.115307705 & 1.842406489 & -2.423092765 \\
\hline 6 & 4.049895198 & 3.760034252 & -1.416489138 \\
\hline 1 & 4.993846273 & 4.106898821 & -1.836817952 \\
\hline 6 & 3.324252457 & 4.586376257 & -0.541085290 \\
\hline 1 & 3.703779391 & 5.574379262 & -0.280686573 \\
\hline 6 & 2.107027913 & 4.147413956 & 0.000560286 \\
\hline 1 & 1.556546755 & 4.807409180 & 0.669919516 \\
\hline 8 & -1.306823183 & -1.974724231 & -0.494110154 \\
\hline 6 & -0.395187601 & -1.915615430 & -1.385531701 \\
\hline 1 & 0.638669623 & -2.211682076 & -1.126064912 \\
\hline 6 & -0.724274096 & -1.727514217 & -2.861129835 \\
\hline 1 & -1.778221063 & -1.991148723 & -3.016270934 \\
\hline 1 & -0.107248762 & -2.415543658 & -3.458033328 \\
\hline 6 & -0.443326045 & -0.269909335 & -3.317024345 \\
\hline 1 & -0.813763025 & -0.129760484 & -4.347562207 \\
\hline 1 & 0.642211654 & -0.103605233 & -3.364938489 \\
\hline 6 & -1.066746648 & 0.790185812 & -2.420091849 \\
\hline 1 & -0.654807822 & 1.790718294 & -2.563479169 \\
\hline 6 & -2.258306465 & 0.678134553 & -1.715241572 \\
\hline 1 & -2.863984053 & -0.226881992 & -1.748872737 \\
\hline 1 & -2.756556430 & 1.573264394 & -1.346238052 \\
\hline \multicolumn{4}{|r|}{ 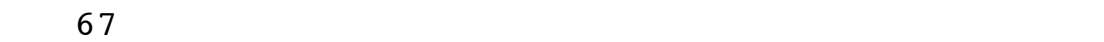 } \\
\hline & T.E. = & -6794.1094260168 & \\
\hline 45 & -0.124773604 & 0.081241829 & -2.184696527 \\
\hline 15 & -1.914109252 & -1.416039043 & -2.500155779 \\
\hline 6 & -3.512314289 & -0.425889338 & -2.568339145 \\
\hline 1 & -4.295114279 & -0.992106171 & -3.088876508 \\
\hline 1 & -3.855242025 & -0.239573307 & -1.543558034 \\
\hline 6 & -3.212469462 & 0.907665138 & -3.283087832 \\
\hline 1 & -2.995185490 & 0.733502007 & -4.344194328 \\
\hline 1 & -4.064515154 & 1.597392205 & -3.223962188 \\
\hline 15 & -1.690356220 & 1.689228010 & -2.491526669 \\
\hline 6 & -1.871726083 & -2.306261997 & -4.124573608 \\
\hline 6 & -0.856142318 & -2.016056657 & -5.059702665 \\
\hline 1 & -0.085554584 & -1.286332697 & -4.809812158 \\
\hline 6 & -0.833582528 & -2.661003086 & -6.307575970 \\
\hline 1 & -0.040829326 & -2.433315312 & -7.020334642 \\
\hline 6 & -1.825821648 & -3.598113585 & -6.632631573 \\
\hline 1 & -1.808090902 & -4.100143641 & -7.599838352 \\
\hline 6 & -2.841997689 & -3.892750611 & -5.706742474 \\
\hline 1 & -3.612510109 & -4.623289052 & -5.952879664 \\
\hline 6 & -2.866904223 & -3.253786585 & -4.458875929 \\
\hline 1 & -3.652687495 & -3.505995091 & -3.747478070 \\
\hline 6 & -2.121170854 & -2.728051009 & -1.213961232 \\
\hline
\end{tabular}




\begin{tabular}{|c|c|c|c|}
\hline 6 & -1.443074070 & -3.959543167 & -1.367322945 \\
\hline 1 & -0.863008694 & -4.162617572 & -2.265246792 \\
\hline 6 & -1.516123253 & -4.938790601 & -0.365985297 \\
\hline 1 & -0.992537684 & -5.884789202 & -0.502066576 \\
\hline 6 & -2.260861595 & -4.704424595 & 0.800668959 \\
\hline 1 & -2.321824247 & -5.468954783 & 1.575003014 \\
\hline 6 & -2.929386213 & -3.480532179 & 0.966065116 \\
\hline 1 & -3.510966633 & -3.290732752 & 1.868286150 \\
\hline 6 & -2.855510038 & -2.493641673 & -0.028650107 \\
\hline 1 & -3.373165519 & -1.549984056 & 0.132417845 \\
\hline 6 & -1.246241184 & 3.128278706 & -3.567093701 \\
\hline 6 & -1.218969508 & 2.979979350 & -4.973620479 \\
\hline 1 & -1.498762224 & 2.036738751 & -5.439854730 \\
\hline 6 & -0.825354581 & 4.047504462 & -5.793684772 \\
\hline 1 & -0.821958012 & 3.921587223 & -6.876331932 \\
\hline 6 & -0.436606962 & 5.271261706 & -5.223636610 \\
\hline 1 & -0.130072570 & 6.099384384 & -5.862378733 \\
\hline 6 & -0.439892813 & 5.421513839 & -3.828208731 \\
\hline 1 & -0.135696188 & 6.366407102 & -3.377856867 \\
\hline 6 & -0.838618000 & 4.358189042 & -3.002342835 \\
\hline 1 & -0.841278506 & 4.496661480 & -1.922687196 \\
\hline 6 & -2.372421522 & 2.424938270 & -0.927314181 \\
\hline 6 & -1.922882510 & 1.966618955 & 0.328659137 \\
\hline 1 & -1.146479710 & 1.203551819 & 0.374662636 \\
\hline 6 & -2.475139088 & 2.480371502 & 1.514298854 \\
\hline 1 & -2.116461401 & 2.118498437 & 2.478271440 \\
\hline 6 & -3.483711908 & 3.453899688 & 1.457641275 \\
\hline 1 & -3.912014676 & 3.853013226 & 2.377121273 \\
\hline 6 & -3.940600363 & 3.914443229 & 0.210467088 \\
\hline 1 & -4.723318235 & 4.671328855 & 0.158541781 \\
\hline 6 & -3.390739809 & 3.405840548 & -0.975307325 \\
\hline 1 & -3.751168102 & 3.785776660 & -1.930808053 \\
\hline 8 & 2.160315394 & -3.143729221 & -1.704617754 \\
\hline 6 & 2.051670497 & -1.943738675 & -1.596906672 \\
\hline 1 & 1.041539817 & -1.471142937 & -1.934460607 \\
\hline 6 & 3.077799282 & -0.957169556 & -1.077781077 \\
\hline 1 & 3.697333210 & -0.661741271 & -1.940945083 \\
\hline 1 & 3.745641660 & -1.497140430 & -0.393420771 \\
\hline 6 & 2.464724916 & 0.285843451 & -0.396126976 \\
\hline 1 & 3.298523557 & 0.882833360 & 0.012460597 \\
\hline 1 & 1.875797727 & -0.025275856 & 0.480574920 \\
\hline 6 & 1.630886532 & 1.227226246 & -1.262766372 \\
\hline 1 & 1.208333230 & 2.061785151 & -0.699631332 \\
\hline 6 & 1.687633814 & 1.345924425 & -2.651609074 \\
\hline 1 & 2.328891217 & 0.704599675 & -3.259248344 \\
\hline 1 & 1.363060234 & 2.261947135 & -3.138845227 \\
\hline \multicolumn{4}{|r|}{ - } \\
\hline $2 \mathrm{C}$ & \multicolumn{3}{|c|}{ T.E. $=-6794.1060325525$} \\
\hline 45 & 0.122718801 & 0.087373996 & -1.742639436 \\
\hline 15 & -1.560337771 & -1.511249759 & -2.147777868 \\
\hline 6 & -3.209736945 & -0.618479155 & -2.032706787 \\
\hline 1 & -4.012695747 & -1.287899467 & -2.368322527 \\
\hline 1 & -3.399375522 & -0.376567323 & -0.979330211 \\
\hline 6 & -3.179921259 & 0.671341663 & -2.884371339 \\
\hline 1 & -3.050796030 & 0.420608130 & -3.944039581 \\
\hline
\end{tabular}




\begin{tabular}{|c|c|c|c|}
\hline 1 & -4.125344190 & 1.220132697 & -2.78561567 \\
\hline 15 & -1.739568452 & 1.757865939 & -2.33419081 \\
\hline 6 & -1.572735691 & -2.181166855 & -3.8762270 \\
\hline 6 & -0.592043142 & -1.800749528 & -4.8151640 \\
\hline 1 & 0.233439328 & -1.157535334 & -4.5197098 \\
\hline 6 & -0.646452934 & -2.279825749 & -6.1343948 \\
\hline 1 & 0.124113704 & -1.984765390 & -6.8464171 \\
\hline 6 & -1.677733957 & -3.145333227 & -6.5277190 \\
\hline 1 & -1.717251569 & -3.521074295 & -7.5499024 \\
\hline 6 & -2.657141090 & -3.533715866 & -5.5974100 \\
\hline 1 & -3.456341632 & -4.212757361 & -5.8942178 \\
\hline 6 & -2.608073827 & -3.056672903 & -4.2799918 \\
\hline 1 & -3.366632459 & -3.385099013 & -3.5706385 \\
\hline 6 & -1.677783617 & -2.974315171 & -1.02859 \\
\hline 6 & -1.017732169 & -4.175197499 & -1.3700311 \\
\hline 1 & -0.477246814 & -4.257822265 & -2.3102480 \\
\hline 6 & -1.052287523 & -5.271861867 & -0.4950576 \\
\hline 1 & -0.541158222 & -6.193813466 & -0.7717075 \\
\hline 6 & -1.740726728 & -5.184872761 & 0.7251007 \\
\hline 1 & -1.768711618 & -6.040354329 & 1.3998173 \\
\hline 6 & -2.392364797 & -3.990885922 & 1.0741296 \\
\hline 1 & -2.930168750 & -3.915626473 & 2.0192201 \\
\hline 6 & -2.357775544 & -2.888246696 & 0.2073094 \\
\hline 1 & -2.864367788 & -1.972472877 & 0.5071434 \\
\hline 6 & -1.449849720 & 2.975716056 & -3.7018403 \\
\hline 6 & -1.538947892 & 2.590202198 & -5.0591785 \\
\hline 1 & -1.871268453 & 1.591541013 & -5.3368221 \\
\hline 6 & -1.203293582 & 3.492986757 & -6.0806650 \\
\hline 1 & -1.292110215 & 3.182040911 & -7.1216562 \\
\hline 6 & -0.759153498 & 4.786823250 & -5.7660404 \\
\hline 1 & -0.500590389 & 5.485965736 & -6.5609013 \\
\hline 6 & -0.652743510 & 5.175337758 & -4.4211275 \\
\hline 1 & -0.312090530 & 6.179013848 & -4.16647 \\
\hline 6 & -0.992458783 & 4.279202702 & -3.3956994 \\
\hline 1 & -0.917324979 & 4.607082065 & -2.3597986 \\
\hline 6 & -2.491599740 & 2.745097545 & -0.9506458 \\
\hline 6 & -2.065055673 & 2.536697643 & 0.3775257 \\
\hline 1 & -1.270862538 & 1.822205946 & 0.5850755 \\
\hline 6 & -2.664842008 & 3.235147217 & 1.4394535 \\
\hline 1 & -2.323824204 & 3.064504896 & 2.4607787 \\
\hline 6 & -3.697754185 & 4.149087286 & 1.1840942 \\
\hline 1 & -4.162881346 & 4.693647354 & 2.0056496 \\
\hline 6 & -4.132762275 & 4.361405746 & -0.1357153 \\
\hline 1 & -4.935306643 & 5.070520501 & -0.3392107 \\
\hline 6 & -3.537309400 & 3.665553752 & -1.1973028 \\
\hline 1 & -3.881653431 & 3.851086595 & -2.2141047 \\
\hline 8 & 1.712161022 & -2.213720418 & -2.4359923 \\
\hline 6 & 1.672027140 & -1.268527210 & -1.6647330 \\
\hline 1 & -0.164849466 & -0.377533142 & -0.3003574 \\
\hline 6 & 2.896982405 & -0.903331661 & -0.7859170 \\
\hline 1 & 3.694712231 & -0.624846044 & -1.4916648 \\
\hline 1 & 3.244048797 & -1.812996958 & -0.2767571 \\
\hline 6 & 2.576384293 & 0.230355301 & 0.1987109 \\
\hline 1 & 3.510097931 & 0.700960710 & 0.5509323 \\
\hline 1 & 2.079927388 & -0.168811081 & $1.093143 \xi$ \\
\hline
\end{tabular}




\begin{tabular}{|c|c|c|c|}
\hline 6 & 1.697721412 & 1.307429931 & -0.411399538 \\
\hline 1 & 1.133757438 & 1.912156323 & 0.300172452 \\
\hline 6 & 1.751903169 & 1.749226893 & -1.724477257 \\
\hline 1 & 2.485128786 & 1.353699981 & -2.430321168 \\
\hline 1 & 1.293355102 & 2.694102282 & -2.006629796 \\
\hline \multicolumn{4}{|c|}{ 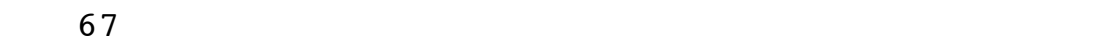 } \\
\hline & $\mathrm{T} . \mathrm{E} .=-6$ & 94.1072318743 & \\
\hline 45 & 1.228331351 & -6.207899752 & 0.391523727 \\
\hline 15 & -0.415916482 & -7.799703362 & -0.150162893 \\
\hline 6 & -2.063728405 & -6.902913369 & -0.065623153 \\
\hline 1 & -2.860129695 & -7.560234073 & -0.438124416 \\
\hline 1 & -2.281097752 & -6.680993369 & 0.986855932 \\
\hline 6 & -1.999576706 & -5.596074578 & -0.888498997 \\
\hline 1 & -1.852488393 & -5.823083168 & -1.951069567 \\
\hline 1 & -2.940418635 & -5.039034609 & -0.795665552 \\
\hline 15 & -0.561261398 & -4.531096443 & -0.286902247 \\
\hline 6 & -0.384984212 & -8.457916331 & -1.882838938 \\
\hline 6 & 0.634092601 & -8.096634600 & -2.787975800 \\
\hline 1 & 1.462576370 & -7.472338011 & -2.464103476 \\
\hline 6 & 0.612813779 & -8.575955485 & -4.108556856 \\
\hline 1 & 1.413040513 & -8.297152873 & -4.793993323 \\
\hline 6 & -0.423622060 & -9.417446741 & -4.537939591 \\
\hline 1 & -0.437386471 & -9.790744605 & -5.561696585 \\
\hline 6 & -1.441325648 & -9.786034119 & $-3.64 c$ \\
\hline 1 & -2.245396072 & -10.446545960 & -3.965173725 \\
\hline 6 & -1.424040199 & -9.312987161 & -2.321663492 \\
\hline 1 & -2.211669307 & -9.627629825 & -1.638163192 \\
\hline 6 & -0.563890639 & -9.280649130 & 0.945170322 \\
\hline 6 & 0.106034821 & -10.475785371 & 0.601403507 \\
\hline 1 & 0.660958661 & -10.547424697 & -0.332091962 \\
\hline 6 & 0.056229136 & -11.585719535 & 1.459057686 \\
\hline 1 & 0.573858403 & -12.502949682 & 1.178768970 \\
\hline 6 & -0.657833156 & -11.518073980 & 2.665797257 \\
\hline 1 & -0.697941744 & -12.383257438 & 3.327 \\
\hline 6 & -1.320246410 & -10.330804873 & 3.017 \\
\hline 1 & -1.879267347 & -10.270653561 & 3.951008192 \\
\hline 6 & -1.269982310 & -9.215278354 & 2.167286911 \\
\hline 1 & -1.786507895 & -8.305683875 & 2.469103981 \\
\hline 6 & -0.185245100 & -3.350200166 & -1.666769104 \\
\hline 6 & 0.190152557 & -2.016140391 & -1.384995905 \\
\hline 1 & 0.153272217 & -1.637274679 & -0.365053462 \\
\hline 6 & 0.591292121 & -1.154610102 & -2.418169571 \\
\hline 1 & 0.867849434 & -0.126681927 & -2.182816036 \\
\hline 6 & 0.629020862 & -1.608772480 & -3.745698149 \\
\hline 1 & 0.936826631 & -0.937364453 & -4.546945260 \\
\hline 6 & 0.267106849 & -2.933951651 & -4.035420341 \\
\hline 1 & 0.291167590 & -3.295847254 & -5.063293102 \\
\hline 6 & -0.127860877 & -3.801994303 & -3.005722858 \\
\hline 1 & -0.390451640 & -4.827130029 & -3.262062138 \\
\hline 6 & -1.348624844 & -3.498822918 & 1.042322366 \\
\hline 6 & -0.921172883 & -3.622195370 & 2.380650819 \\
\hline 1 & -0.110663141 & -4.303868108 & 2.630145988 \\
\hline 6 & -1.536640205 & -2.876195608 & 3.400426400 \\
\hline 1 & -1.194080241 & -2.981831431 & 4.429930828 \\
\hline 6 & -2.586024134 & -1.998080182 & 3.09293491 \\
\hline
\end{tabular}




\begin{tabular}{|c|c|c|c|}
\hline 1 & -3.062994219 & -1.417029615 & 3.882080695 \\
\hline 6 & -3.021273759 & -1.868132453 & 1.762466353 \\
\hline 1 & -3.836180754 & -1.186812693 & 1.517782210 \\
\hline 6 & -2.409644120 & -2.611503424 & 0.743223736 \\
\hline 1 & -2.753807632 & -2.487285700 & -0.283007575 \\
\hline 8 & 3.033069672 & -8.191384602 & -0.498872931 \\
\hline 6 & 2.700796644 & -7.638360948 & 0.539068498 \\
\hline 1 & 0.697261222 & -6.536875822 & 1.795750704 \\
\hline 6 & 3.522747806 & -7.690237750 & 1.830557002 \\
\hline 1 & 4.347209761 & -8.412697862 & 1.752694129 \\
\hline 1 & 2.860345101 & -7.973814162 & 2.660428848 \\
\hline 6 & 4.030190202 & -6.232244130 & 2.009117554 \\
\hline 1 & 4.817659678 & -6.036338341 & 1.268618960 \\
\hline 1 & 4.475907836 & -6.110170070 & 3.006599581 \\
\hline 6 & 2.901594986 & -5.221572648 & 1.844979308 \\
\hline 1 & 2.339526578 & -4.987009890 & 2.7501 \\
\hline 6 & 2.719925439 & -4.413261012 & 0.734780559 \\
\hline 1 & 3.403421096 & -4.475761718 & -0.115774737 \\
\hline 1 & 2.116084780 & -3.511756232 & 0.793613328 \\
\hline \multicolumn{4}{|c|}{67} \\
\hline & T.E. $=-679$ & 1187127523 & \\
\hline 45 & -1.231279710 & -3.704685977 & -1.072863520 \\
\hline 15 & -3.197065565 & -4.985718419 & -1.408436498 \\
\hline 6 & -4.625241229 & -3.778583023 & -1.675 \\
\hline 1 & -5.409973557 & -4.249395866 & -2.277770705 \\
\hline 1 & -5.062526507 & -3.588689255 & -0.687206757 \\
\hline 6 & -4.165150145 & -2.459043141 & -2.331278044 \\
\hline 1 & -3.815021429 & -2.643788826 & -3.353003321 \\
\hline 1 & -4.999266310 & -1.746934357 & -2.381801433 \\
\hline 15 & -2.727626820 & -1.727403074 & -1.367727370 \\
\hline 6 & -3.232952557 & -6.223021669 & -2.785591250 \\
\hline 6 & -2.058721651 & -6.943283915 & -3.095917626 \\
\hline 1 & -1.140468837 & -6.747434784 & -2.546437613 \\
\hline 6 & -2.063610824 & -7.919745129 & -4.102 \\
\hline 1 & -1.147863911 & -8.465969648 & -4.328322024 \\
\hline 6 & -3.239991082 & -8.187758916 & -4.819803345 \\
\hline 1 & -3.241804371 & -8.938788127 & -5.609483580 \\
\hline 6 & -4.414882188 & -7.484440622 & -4.514021032 \\
\hline 1 & -5.335986852 & -7.691364146 & -5.058463384 \\
\hline 6 & -4.415598223 & -6.512994430 & -3.500921153 \\
\hline 1 & -5.349465732 & -6.002999238 & -3.277113848 \\
\hline 6 & -3.66 & -5.9356322 & 0.115939559 \\
\hline 6 & -3.665845635 & -7.347017095 & 0.134263218 \\
\hline 1 & -3.427227843 & -7.910220954 & -0.764652418 \\
\hline 6 & -3.985393718 & -8.040987159 & 1.311918107 \\
\hline 1 & -3.987754866 & -9.130974002 & 1.306714307 \\
\hline 6 & -4.305895022 & -7.340879964 & 2.484768611 \\
\hline 1 & -4.557650902 & -7.883494107 & 3.395621471 \\
\hline 6 & -4.304891517 & -5.936684753 & 2.477593850 \\
\hline 1 & -4.557144792 & -5.383338633 & 3.382103842 \\
\hline 6 & -3.984279708 & -5.237627857 & 1.304764082 \\
\hline 1 & -3.992217435 & -4.149255055 & 1.327702978 \\
\hline 6 & -2.161041662 & -0.246596710 & -2.328242824 \\
\hline 6 & -1.674313853 & 0.889257659 & -1.640169505 \\
\hline 1 & -1.700150797 & 0.927983891 & -0.55223103 \\
\hline
\end{tabular}




\begin{tabular}{|c|c|c|c|c|}
\hline 6 & & -1.175836224 & 1.992255224 & -2.350074523 \\
\hline 1 & & -0.813917877 & 2.863292202 & -1.803777110 \\
\hline 6 & & -1.154129940 & 1.979362548 & -3.753828030 \\
\hline 1 & & -0.773702174 & 2.839723209 & -4.303943515 \\
\hline 6 & & -1.629202928 & 0.853830373 & -4.444742251 \\
\hline 1 & & -1.618534684 & 0.834920453 & -5.534579602 \\
\hline 6 & & -2.123323144 & -0.256106690 & -3.741281925 \\
\hline 1 & & -2.470892586 & -1.118732338 & -4.304190750 \\
\hline 6 & & -3.528990160 & -1.009526975 & 0.146745569 \\
\hline 6 & & -3.064031928 & -1.356827983 & 1.432678672 \\
\hline 1 & & -2.226553530 & -2.043276112 & 1.544605797 \\
\hline 6 & & -3.667834644 & -0.821315365 & 2.583452892 \\
\hline 1 & & -3.292363768 & -1.096008527 & 3.569204359 \\
\hline 6 & & -4.746479565 & 0.066804883 & 2.461330803 \\
\hline 1 & & -5.215793751 & 0.483308278 & 3.352481487 \\
\hline 6 & & -5.216503930 & 0.423334693 & 1.185423149 \\
\hline 1 & & -6.049243614 & 1.119299258 & 1.083871387 \\
\hline 6 & & -4.611501856 & -0.105547297 & 0.036547623 \\
\hline 1 & & -4.977181972 & 0.202965209 & -0.942043873 \\
\hline 8 & & -1.343090449 & -3.641083964 & -3.937276471 \\
\hline 6 & & -0.604467547 & -3.782727120 & -2.987573089 \\
\hline 1 & & -0.607678134 & -5.164831973 & -0.884086053 \\
\hline 6 & & 0.915796374 & -4.043246283 & -3.120167018 \\
\hline 1 & & 1.218581065 & -3.786138995 & -4.144390124 \\
\hline 1 & & 1.043505561 & -5.127184111 & -2.988334023 \\
\hline 6 & & 1.717833743 & -3.266204097 & -2.056282327 \\
\hline 1 & & 1.775044580 & -2.209724793 & -2.352692658 \\
\hline 1 & & 2.747406907 & -3.648361197 & -2.015685260 \\
\hline 6 & & 1.067186014 & -3.366748001 & -0.682499555 \\
\hline 1 & & 1.365124011 & -4.196818671 & -0.043490768 \\
\hline 6 & & 0.355443310 & -2.306494974 & -0.137678295 \\
\hline 1 & & 0.339724117 & -1.335577893 & -0.630140721 \\
\hline 1 & & 0.092392895 & -2.312420254 & 0.921860111 \\
\hline & 67 & & & \\
\hline $2 a$ & +5.2 & \multicolumn{3}{|c|}{ T.E. $=-6794.1219825368$} \\
\hline 45 & & -1.208842525 & -3.645029478 & -1.105127895 \\
\hline 15 & & -3.180606606 & -4.919228943 & -1.425477670 \\
\hline 6 & & -4.609793063 & -3.726030655 & -1.766077879 \\
\hline 1 & & -5.357161116 & -4.211555558 & -2.406309686 \\
\hline 1 & & -5.082648622 & -3.546316876 & -0.791893659 \\
\hline 6 & & -4.145321647 & -2.390178121 & -2.387063307 \\
\hline 1 & & -3.793741501 & -2.538956440 & -3.413797355 \\
\hline 1 & & -4.977547177 & -1.673937159 & -2.411457054 \\
\hline 15 & & -2.710361907 & -1.681134982 & -1.399473677 \\
\hline 6 & & -3.203254881 & -6.191279323 & -2.768636010 \\
\hline 6 & & -3.718069991 & -5.916347775 & -4.052969184 \\
\hline 1 & & -4.137972941 & -4.941904412 & -4.291574958 \\
\hline 6 & & -3.690659244 & -6.901545070 & -5.052771782 \\
\hline 1 & & -4.094578129 & -6.676562624 & -6.039685177 \\
\hline 6 & & -3.144840805 & -8.165797692 & -4.784525867 \\
\hline 1 & & -3.127305703 & -8.929896078 & -5.561437132 \\
\hline 6 & & -2.623171147 & -8.444132726 & -3.510162573 \\
\hline 1 & & -2.202165196 & -9.425705992 & -3.292678361 \\
\hline 6 & & -2.647033138 & -7.464032389 & -2.508046842 \\
\hline 1 & & -2.245517050 & -7.699609951 & -1.523589991 \\
\hline
\end{tabular}




\begin{tabular}{|c|c|c|c|}
\hline 6 & -3.713965592 & -5.838890457 & 0.090655744 \\
\hline 6 & -2.850490006 & -5.971090882 & 1.197541045 \\
\hline 1 & -1.847247538 & -5.547757349 & 1.160267166 \\
\hline 6 & -3.268224019 & -6.660941570 & 2.348114323 \\
\hline 1 & -2.588614887 & -6.760091028 & 3.194749667 \\
\hline 6 & -4.550810265 & -7.226189468 & 2.402152219 \\
\hline 1 & -4.873848781 & -7.764785493 & 3.292805667 \\
\hline 6 & -5.415980216 & -7.104217726 & 1.301229991 \\
\hline 1 & -6.410100586 & -7.549717151 & 1.334221607 \\
\hline 6 & -5.001975756 & -6.420057043 & 0.150028921 \\
\hline 1 & -5.681397059 & -6.354028858 & -0.698968177 \\
\hline 6 & -2.148239099 & -0.172222366 & -2.313793760 \\
\hline 6 & -2.115609306 & -0.139042199 & -3.726473543 \\
\hline 1 & -2.467243256 & -0.983746778 & -4.31401469 \\
\hline 6 & -1.619787047 & 0.989626995 & -4.398195934 \\
\hline 1 & -1.611943278 & 1.003579024 & -5.488104593 \\
\hline 6 & -1.138187699 & 2.091802797 & -3.674980394 \\
\hline 1 & -0.756961925 & 2.967127724 & -4.200293795 \\
\hline 6 & -1.152997273 & 2.061733988 & -2.271327963 \\
\hline 1 & -0.784044500 & 2.913865658 & -1.700430204 \\
\hline 6 & -1.651620565 & 0.938965671 & -1.593156206 \\
\hline 1 & -1.668640519 & 0.941862859 & -0.504317577 \\
\hline 6 & -3.527213137 & -1.037125123 & 0.136167159 \\
\hline 6 & -3.190744005 & -1.576310689 & 1.395215215 \\
\hline 1 & -2.429334933 & -2.351532209 & 1.474965422 \\
\hline 6 & -3.831612030 & -1.127258563 & 2.561992457 \\
\hline 1 & -3.559946197 & -1.554865268 & 3.526963935 \\
\hline 6 & -4.816306624 & -0.131744770 & 3117280 \\
\hline 1 & -5.313254130 & 0.218893498 & 3.3 \\
\hline 6 & -5.159016280 & 0.414672203 & 1.234046079 \\
\hline 1 & -5.920323587 & 1.191864118 & 1.166175981 \\
\hline 6 & -4.521066331 & -0.032493628 & 0.068617499 \\
\hline 1 & -4.790125432 & 0.414569254 & -0.887701161 \\
\hline 8 & -1.304119780 & -3.616329281 & -3.964653997 \\
\hline 6 & -0.580871915 & -3.808439194 & -3.012909742 \\
\hline 1 & -0.602019955 & -5.110448051 & -0.893979868 \\
\hline 6 & 0.912164520 & -4.191003 & -3.121678388 \\
\hline 1 & 1.24 & -4 & -4 \\
\hline 1 & 0.953356650 & -5.273279277 & -2.933806214 \\
\hline 6 & 1.762892575 & -3.422239373 & -2.086064866 \\
\hline 1 & 1.937180702 & -2.401607616 & -2.454020020 \\
\hline 1 & 2.746051383 & -3.902719132 & -1.989200576 \\
\hline 6 & 1.078382879 & -3.350790334 & -0.723416261 \\
\hline 1 & 1.346595079 & -4.106289383 & 0.013714602 \\
\hline 6 & 0.398638762 & -2.207930012 & -0.311986947 \\
\hline 1 & 0.420126179 & -1.301438303 & -0.915088532 \\
\hline 1 & 0.128769951 & -2.075424052 & 0.737620574 \\
\hline \multicolumn{4}{|c|}{ - } \\
\hline & T.E. = & -6794.116272 & \\
\hline 45 & -0.467133200 & 0.168467900 & -0.318781400 \\
\hline 15 & 0.447583500 & -0.578496800 & 1.732281500 \\
\hline 6 & 1.231769900 & 0.899195500 & 2.589181300 \\
\hline 1 & 1.312600900 & 0.721222700 & 3.668707600 \\
\hline 1 & 2.245292500 & 1.039120000 & 2.194906500 \\
\hline 6 & 0.365814000 & 2.139517200 & 2.287604400 \\
\hline
\end{tabular}




\begin{tabular}{|c|c|c|c|}
\hline 1 & -0.606727500 & 2.059996500 & 2.788733200 \\
\hline 1 & 0.849744200 & 3.061262200 & 2.635233900 \\
\hline 15 & 0.070369300 & 2.227638700 & 0.427959200 \\
\hline 6 & -0.839762400 & -1.156462000 & 2.933454200 \\
\hline 6 & -2.199751500 & -1.169945600 & 2.559233700 \\
\hline 1 & -2.488370100 & -0.877324400 & 1.550312400 \\
\hline 6 & -3.184441300 & -1.566886200 & 3.478596900 \\
\hline 1 & -4.232049000 & -1.578163600 & 3.176724600 \\
\hline 6 & -2.820640800 & -1.954081000 & 4.776955000 \\
\hline 1 & -3.585676200 & -2.263822300 & 5.488723000 \\
\hline 6 & -1.466992800 & -1.947947600 & 5.156891600 \\
\hline 1 & -1.179574100 & -2.253536200 & 6.162899700 \\
\hline 6 & -0.479951800 & -1.554870500 & 4.242012400 \\
\hline 1 & 0.565537300 & -1.573154800 & 4.547987700 \\
\hline 6 & 1.721838200 & -1.919871900 & 1.683184700 \\
\hline 6 & 1.320376900 & -3.271516900 & 1.788238900 \\
\hline 1 & 0.273103400 & -3.521560200 & 1.945937400 \\
\hline 6 & 2.269167900 & -4.302443400 & 1.705308900 \\
\hline 1 & 1.944612800 & -5.338508500 & 1.804076800 \\
\hline 6 & 3.626710600 & -4.002413600 & 1.509327400 \\
\hline 1 & 4.362972400 & -4.804716300 & 1.459289500 \\
\hline 6 & 4.032185300 & -2.663669100 & 1.384145800 \\
\hline 1 & 5.084271600 & -2.421602300 & 1.232370700 \\
\hline 6 & 3.087627900 & -1.628124100 & 1.463978200 \\
\hline 1 & 3.430954300 & -0.600347300 & 1.361643900 \\
\hline 6 & -1.186571800 & 3.568435700 & 0.183196900 \\
\hline 6 & -2.336184400 & 3.618590300 & 1.006181900 \\
\hline 1 & -2.470358700 & 2.906183800 & 1.818548400 \\
\hline 6 & -3.327898000 & 4.586509600 & 0.786769700 \\
\hline 1 & -4.201473200 & 4.618829900 & 1.437949700 \\
\hline 6 & -3.197629900 & 5.506572800 & -0.266731400 \\
\hline 1 & -3.969113400 & 6.257471300 & -0.436114600 \\
\hline 6 & -2.070254200 & 5.452977500 & -1.100787300 \\
\hline 1 & -1.961516500 & 6.162773600 & -1.920995700 \\
\hline 6 & -1.071284900 & 4.490898000 & -0.880961200 \\
\hline 1 & -0.198718100 & 4.476249400 & -1.532218800 \\
\hline 6 & 1.674860900 & 2.939949000 & -0.190664700 \\
\hline 6 & 2.508126100 & 2.180833500 & -1.038229100 \\
\hline 1 & 2.183835200 & 1.191326300 & -1.357888000 \\
\hline 6 & 3.750158400 & 2.686081100 & -1.458955600 \\
\hline 1 & 4.384089600 & 2.088701200 & -2.114820700 \\
\hline 6 & 4.173796700 & 3.954803500 & -1.035065300 \\
\hline 1 & 5.136482600 & 4.348628200 & -1.360846900 \\
\hline 6 & 3.352543500 & 4.717283900 & -0.186473200 \\
\hline 1 & 3.677025700 & 5.702517400 & 0.148364200 \\
\hline 6 & 2.111655000 & 4.216617100 & 0.233573700 \\
\hline 1 & 1.486911700 & 4.828740000 & 0.883349800 \\
\hline 8 & -0.994689500 & -2.020348000 & -0.792013500 \\
\hline 6 & -0.281683800 & -2.238707600 & -1.796941900 \\
\hline 1 & 0.613409300 & -2.884977700 & -1.682079600 \\
\hline 6 & -0.609886100 & -1.738199300 & -3.189653300 \\
\hline 1 & -1.697897800 & -1.797509500 & -3.329705400 \\
\hline 1 & -0.129573100 & -2.397780700 & -3.925913700 \\
\hline 6 & -0.119569200 & -0.279776500 & -3.436668500 \\
\hline 1 & -0.352156700 & -0.016889300 & -4.483764700 \\
\hline
\end{tabular}




\begin{tabular}{|c|c|c|c|}
\hline 1 & 0.977350100 & -0.244027200 & -3.359230500 \\
\hline 6 & -0.725174600 & 0.788347900 & -2.532667100 \\
\hline 1 & -0.263864800 & 1.768112000 & -2.673494600 \\
\hline 6 & -1.965588800 & 0.742853000 & -1.900774100 \\
\hline 1 & -2.607342400 & -0.135442700 & -1.962694000 \\
\hline 1 & -2.451889200 & 1.661985000 & -1.581975800 \\
\hline \multicolumn{4}{|c|}{67} \\
\hline \multicolumn{4}{|c|}{ TS2pi-2sigma +15.8 T.E. $=-6794.104909$} \\
\hline 45 & -0.582691500 & 0.145230000 & -0.184861800 \\
\hline 15 & 0.408390800 & -0.549661800 & 1.825425100 \\
\hline 6 & 1.320986900 & 0.917858500 & 2.561905400 \\
\hline 1 & 1.485081200 & 0.774201100 & 3.637316100 \\
\hline 1 & 2.301813600 & 1.001147600 & 2.078564600 \\
\hline 6 & 0.479548800 & 2.180198800 & 2.283864500 \\
\hline 1 & -0.447469200 & 2.162083400 & 2.869923800 \\
\hline 1 & 1.027068900 & 3.094434300 & 2.546879900 \\
\hline 15 & 0.026211400 & 2.209558300 & 0.453656700 \\
\hline 6 & -0.807836000 & -1.032901600 & 3.134893100 \\
\hline 6 & -2.193202300 & -0.982408600 & 2.875713900 \\
\hline 1 & -2.550017300 & -0.690632000 & 1.888761000 \\
\hline 6 & -3.115075400 & -1.314185300 & 3.882692600 \\
\hline 1 & -4.183552500 & -1.277623900 & 3.669621400 \\
\hline 6 & -2.662034300 & -1.695945300 & 5.154451500 \\
\hline 1 & -3.377581000 & -1.951405400 & 5.935809800 \\
\hline 6 & -1.282513500 & -1.754576400 & 5.418646500 \\
\hline 1 & -0.924812700 & -2.058898300 & 6.402247200 \\
\hline 6 & -0.358063600 & -1.428503400 & 4.416253300 \\
\hline 1 & 0.707316600 & -1.497565700 & 4.633576000 \\
\hline 6 & 1.602487500 & -1.955292900 & 1.706254900 \\
\hline 6 & 1.131937000 & -3.281552400 & 1.849532900 \\
\hline 1 & 0.087666300 & -3.472132800 & 2.088135400 \\
\hline 6 & 2.009493500 & -4.364475900 & 1.689326200 \\
\hline 1 & 1.634372600 & -5.380649700 & 1.810818100 \\
\hline 6 & 3.361336300 & -4.142596900 & 1.3818 \\
\hline 1 & 4.042315500 & -4.985577000 & 1.265856700 \\
\hline 6 & 3.833248300 & -2.829104700 & 1.223797600 \\
\hline 1 & 4.881208700 & -2.648192400 & 0.984526000 \\
\hline 6 & 2.960557300 & -1.741259700 & 1.375873900 \\
\hline 1 & 3.350687000 & -0.734669300 & 1.237437900 \\
\hline 6 & -1.204257000 & 3.579209200 & 0.259149000 \\
\hline 6 & -2.280816400 & 3.696547600 & 1.169865200 \\
\hline 1 & -2.378578400 & 3.007995800 & 2.007647800 \\
\hline 6 & -3.244894800 & 4.701740500 & 1.005993500 \\
\hline 1 & -4.060236700 & 4.787857100 & 1.724419700 \\
\hline 6 & -3.161937200 & 5.592340700 & -0.077528200 \\
\hline 1 & -3.912310100 & 6.372834700 & -0.202774100 \\
\hline 6 & -2.110245200 & 5.470625400 & -0.998959800 \\
\hline 1 & -2.040313300 & 6.156061100 & -1.843692800 \\
\hline 6 & -1.138145400 & 4.470292700 & -0.836496800 \\
\hline 1 & -0.324643700 & 4.400307000 & -1.556247700 \\
\hline 6 & 1.591410900 & 2.826819700 & -0.340395900 \\
\hline 6 & 2.302819100 & 2.003078700 & -1.237117200 \\
\hline 1 & 1.904747800 & 1.021080600 & -1.490066200 \\
\hline 6 & 3.518581400 & 2.434751200 & -1.793259100 \\
\hline 1 & 4.056537800 & 1.787685600 & -2.486153600 \\
\hline
\end{tabular}




\begin{tabular}{|c|c|c|c|}
\hline 6 & 4.037130600 & 3.694170600 & -1.456398800 \\
\hline 1 & 4.980253300 & 4.030932200 & -1.886605800 \\
\hline 6 & 3.337086200 & 4.521581400 & -0.561531300 \\
\hline 1 & 3.734412200 & 5.501618600 & -0.297548300 \\
\hline 6 & 2.122001300 & 4.094864200 & -0.005843900 \\
\hline 1 & 1.590529200 & 4.757494800 & 0.676337800 \\
\hline 8 & -1.745687400 & -2.768263200 & -0.578045800 \\
\hline 6 & -1.003877100 & -2.241358400 & -1.402487800 \\
\hline 1 & 0.029591000 & -1.900149300 & -1.080778100 \\
\hline 6 & -1.327129700 & -1.960236600 & -2.857200200 \\
\hline 1 & -2.413001000 & -2.026433000 & -3.004859800 \\
\hline 1 & -0.871063400 & -2.759217300 & -3.465455700 \\
\hline 6 & -0.733353300 & -0.597088000 & -3.291713500 \\
\hline 1 & -1.054690800 & -0.381189600 & -4.324305100 \\
\hline 1 & 0.364244300 & -0.674486800 & -3.337357000 \\
\hline 6 & -1.109825800 & 0.601717100 & -2.426013900 \\
\hline 1 & -0.584133200 & 1.517669200 & -2.702161500 \\
\hline 6 & -2.237716700 & 0.721162700 & -1.618669000 \\
\hline 1 & -2.955461300 & -0.094778700 & -1.513746900 \\
\hline 1 & -2.605876900 & 1.702952000 & -1.329210700 \\
\hline \multicolumn{4}{|c|}{67} \\
\hline \multicolumn{4}{|c|}{ TS2sigma-2C +19.1 T.E. $=-6794.099813$} \\
\hline 45 & -0.067400457 & -0.013349017 & -2.023205090 \\
\hline 15 & -1.893787332 & -1.453121303 & -2.424841671 \\
\hline 6 & -3.465604078 & -0.424106578 & -2.3383 \\
\hline 1 & -4.312947080 & -1.005279584 & -2.724500960 \\
\hline 1 & -3.673338298 & -0.184438270 & -1.288254764 \\
\hline 6 & -3.266742063 & 0.870492521 & -3.156298535 \\
\hline 1 & -3.143802615 & 0.634910352 & -4.220726354 \\
\hline 1 & -4.133835120 & 1.536406542 & -3.057176409 \\
\hline 15 & -1.716083392 & 1.745067347 & -2.546840823 \\
\hline 6 & -1.972866813 & -2.148566887 & -4.142511048 \\
\hline 6 & -0.992835772 & -1.822482032 & -5.102033244 \\
\hline 1 & -0.143391142 & -1.203103391 & -4.822370584 \\
\hline 6 & -1.093513542 & -2.31313 & -6.414 \\
\hline 1 & -0.324281693 & -2.061784968 & -7.144591973 \\
\hline 6 & -2.169860087 & -3.135117695 & -6.779441193 \\
\hline 1 & -2.245207023 & -3.520090911 & -7.796273380 \\
\hline 6 & -3.149390601 & -3.468412919 & -5.827810001 \\
\hline 1 & -3.983912707 & -4.113553469 & -6.102938490 \\
\hline 6 & -3.054686026 & -2.979906248 & -4.516999020 \\
\hline 1 & -3.815201920 & -3.263029003 & -3.790257246 \\
\hline 6 & -2.121550774 & -2.884931861 & -1.281642652 \\
\hline 6 & -1.541269300 & -4.132946343 & -1.601342792 \\
\hline 1 & -1.023070221 & -4.272155506 & -2.547532447 \\
\hline 6 & -1.629351907 & -5.204074797 & -0.699364971 \\
\hline 1 & -1.179287421 & -6.162054349 & -0.959633789 \\
\hline 6 & -2.293309139 & -5.045095923 & 0.527247697 \\
\hline 1 & -2.362460625 & -5.880161706 & 1.224184159 \\
\hline 6 & -2.867667203 & -3.805948789 & 0.853905290 \\
\hline 1 & -3.384553600 & -3.674907189 & 1.804593598 \\
\hline 6 & -2.778200505 & -2.727853779 & -0.040055601 \\
\hline 1 & -3.221926941 & -1.775191296 & 0.243237622 \\
\hline 6 & -1.353342333 & 3.093307515 & -3.760812343 \\
\hline 6 & -1.451783228 & 2.853949843 & -5.150900296 \\
\hline
\end{tabular}




\begin{tabular}{|c|c|c|c|}
\hline 1 & -1.801188247 & 1.895245080 & -5.530256584 \\
\hline 6 & -1.102762161 & 3.855329037 & -6.070194925 \\
\hline 1 & -1.198066571 & 3.659992215 & -7.138118213 \\
\hline 6 & -0.635381618 & 5.100606242 & -5.619342608 \\
\hline 1 & -0.367479577 & 5.876599132 & -6.335758346 \\
\hline 6 & -0.515461969 & 5.340159141 & -4.241688273 \\
\hline 1 & -0.151615651 & 6.302658837 & -3.881478520 \\
\hline 6 & -0.868386818 & 4.345261872 & -3.316517995 \\
\hline 1 & -0.779933473 & 4.556245919 & -2.252011564 \\
\hline 6 & -2.315858253 & 2.594865945 & -1.008768298 \\
\hline 6 & -1.850818921 & 2.184479861 & 0.258536955 \\
\hline 1 & -1.113256508 & 1.386048461 & 0.329963329 \\
\hline 6 & -2.342981358 & 2.788160073 & 1.428122622 \\
\hline 1 & -1.972269259 & 2.462704299 & 2.400503922 \\
\hline 6 & -3.304871457 & 3.805529070 & 1.344652220 \\
\hline 1 & -3.683963835 & 4.276496919 & 2.251464565 \\
\hline 6 & -3.780307709 & 4.215459759 & 0.086712387 \\
\hline 1 & -4.529058375 & 5.004146994 & 0.014017250 \\
\hline 6 & -3.292629993 & 3.615529787 & -1.083267141 \\
\hline 1 & -3.667905675 & 3.954407107 & -2.048120989 \\
\hline 8 & 1.382662457 & -2.478053521 & -2.810593214 \\
\hline 6 & 1.506036263 & -1.496060493 & -2.107279171 \\
\hline 1 & 0.375656425 & -1.199630107 & -1.036039494 \\
\hline 6 & 2.837278267 & -1.077738467 & $-1.451 c$ \\
\hline 1 & 3.512882153 & -0.788032694 & -2.268318682 \\
\hline 1 & 3.276852644 & -1.975109453 & -0.993191064 \\
\hline 6 & 2.635985509 & 0.054725894 & -0.433767825 \\
\hline 1 & 3.610598499 & 0.505229219 & -0.179061423 \\
\hline 1 & 2.231500693 & -0.341959334 & 0.508266923 \\
\hline 6 & 1.715754556 & 1.142951735 & -0.957362695 \\
\hline 1 & 1.276943782 & 1.800217974 & -0.204894020 \\
\hline 6 & 1.641916629 & 1.529434492 & -2.287504004 \\
\hline 1 & 2.270221707 & 1.074721332 & -3.054324409 \\
\hline 1 & 1.202654923 & 2.484181160 & -2.561900830 \\
\hline \multicolumn{4}{|l|}{67} \\
\hline $\mathrm{TS} 2 \mathrm{c}-2 \mathrm{C}$ & +16.5 T.E. & -6794.103722 & \\
\hline 45 & 0.140263500 & 0.107248500 & -1.780878300 \\
\hline 15 & -1.541468600 & -1.512808100 & -2.150058000 \\
\hline 6 & -3.189885900 & -0.622941900 & -1.987085600 \\
\hline 1 & -4.000303300 & -1.294387000 & -2.301039000 \\
\hline 1 & -3.350323400 & -0.380870800 & -0.929017800 \\
\hline 6 & -3.185209500 & 0.666113400 & -2.839838200 \\
\hline 1 & -3.082673100 & 0.415947200 & -3.902528000 \\
\hline 1 & -4.129081200 & 1.211866600 & -2.715928000 \\
\hline 15 & -1.735685600 & 1.759201000 & -2.326935800 \\
\hline 6 & -1.610880200 & -2.197024200 & -3.872801400 \\
\hline 6 & -0.668287700 & -1.817749800 & -4.850506100 \\
\hline 1 & 0.165725200 & -1.171584100 & -4.590973500 \\
\hline 6 & -0.770430200 & -2.307395400 & -6.163440700 \\
\hline 1 & -0.027438100 & -2.014156300 & -6.905183900 \\
\hline 6 & -1.811851100 & -3.179134300 & -6.512530600 \\
\hline 1 & -1.888418400 & -3.561299800 & -7.530277700 \\
\hline 6 & -2.753656300 & -3.565889900 & -5.542992600 \\
\hline 1 & -3.560972200 & -4.249545800 & -5.805087700 \\
\hline 6 & -2.656324000 & -3.081300300 & -4.231302200 \\
\hline
\end{tabular}




\begin{tabular}{|c|c|c|c|}
\hline 1 & -3.386129600 & -3.408127000 & -3.491747100 \\
\hline 6 & -1.626523300 & -2.974127100 & -1.022257100 \\
\hline 6 & -0.966628800 & -4.171928800 & -1.376025700 \\
\hline 1 & -0.449104000 & -4.255056100 & -2.330293600 \\
\hline 6 & -0.985197900 & -5.272448000 & -0.505051900 \\
\hline 1 & -0.479635800 & -6.193723800 & -0.794585900 \\
\hline 6 & -1.657050100 & -5.192336900 & 0.725155000 \\
\hline 1 & -1.676727600 & -6.051862300 & 1.394950100 \\
\hline 6 & -2.306263300 & -4.001347100 & 1.087537100 \\
\hline 1 & -2.832209200 & -3.931191200 & 2.039530700 \\
\hline 6 & -2.288015600 & -2.895550600 & 0.223799300 \\
\hline 1 & -2.796763300 & -1.984208900 & 0.532581000 \\
\hline 6 & -1.459597300 & 2.948579900 & -3.722396400 \\
\hline 6 & -1.528479000 & 2.521844100 & -5.068795200 \\
\hline 1 & -1.837658900 & 1.508643800 & -5.319509600 \\
\hline 6 & -1.198521600 & 3.399828500 & -6.113177900 \\
\hline 1 & -1.270857800 & 3.056959300 & -7.145321800 \\
\hline 6 & -0.778427900 & 4.709573900 & -5.832500800 \\
\hline 1 & -0.520765200 & 5.388300200 & -6.645217100 \\
\hline 6 & -0.691357200 & 5.138834400 & -4.498733500 \\
\hline 1 & -0.367998900 & 6.154580300 & -4.270270300 \\
\hline 6 & -1.025698900 & 4.267222500 & -3.450396600 \\
\hline 1 & -0.961826800 & 4.625155400 & -2.423832600 \\
\hline 6 & -2.470556300 & 2.774723000 & -0.955597800 \\
\hline 6 & -2.010009600 & 2.612909300 & 0.367415300 \\
\hline 1 & -1.201241300 & 1.915879900 & 0.577523400 \\
\hline 6 & -2.588808600 & 3.340982000 & 1.421073100 \\
\hline 1 & -2.219080800 & 3.208171100 & 2.437904900 \\
\hline 6 & -3.635288600 & 4.238303800 & 1.162711400 \\
\hline 1 & -4.080988700 & 4.809265400 & 1.976981400 \\
\hline 6 & -4.104726400 & 4.404251300 & -0.151972500 \\
\hline 1 & -4.916130600 & 5.102224500 & -0.358496300 \\
\hline 6 & -3.529803100 & 3.678960200 & -1.204959700 \\
\hline 1 & -3.897631100 & 3.832437200 & -2.218897900 \\
\hline 8 & 1.945096900 & -1.741694900 & -2.847059000 \\
\hline 6 & 1.719588400 & -1.175064800 & -1.786165700 \\
\hline 1 & -0.193570500 & -0.281205600 & -0.326862500 \\
\hline 6 & 2.698245200 & -1.178764800 & -0.608159100 \\
\hline 1 & 3.686186200 & -1.498144500 & -0.967539800 \\
\hline 1 & 2.336357100 & -1.953165100 & 0.085270700 \\
\hline 6 & 2.730948300 & 0.208868800 & 0.106646000 \\
\hline 1 & 3.743950000 & 0.631505900 & 0.019404800 \\
\hline 1 & 2.539569800 & 0.083524700 & 1.178188000 \\
\hline 6 & 1.747258500 & 1.243126500 & -0.445641800 \\
\hline 1 & 1.182261500 & 1.795566800 & 0.305828900 \\
\hline 6 & 1.758985700 & 1.761655300 & -1.734483900 \\
\hline 1 & 2.488885000 & 1.424480500 & -2.474551300 \\
\hline 1 & 1.272207700 & 2.709213300 & -1.952748600 \\
\hline \multicolumn{4}{|r|}{ 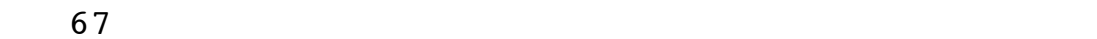 } \\
\hline \multicolumn{4}{|c|}{$\operatorname{TS} 2 \mathrm{C}^{\prime}-2 \mathrm{a}$} \\
\hline 45 & 1.260059100 & -6.139374700 & 0.467504700 \\
\hline 15 & -0.377271200 & -7.777634200 & -0.171501300 \\
\hline 6 & -2.013788400 & -6.844709300 & -0.287556200 \\
\hline 1 & -2.771299400 & -7.515505400 & -0.711926600 \\
\hline 1 & -2.342608800 & -6.573405300 & 0.722974700 \\
\hline
\end{tabular}




\begin{tabular}{|c|c|c|c|}
\hline 6 & -1.852191900 & -5.582729800 & -1.16115370 \\
\hline 1 & -1.538706700 & -5.853905000 & -2.17697050 \\
\hline 1 & -2.803154000 & -5.040052400 & -1.2390014 \\
\hline 15 & -0.543221600 & -4.477248600 & -0.3881209 \\
\hline 6 & -0.363276900 & -8.683633900 & -1.7968366 \\
\hline 6 & 0.628206400 & -8.456675400 & -2.7716130 \\
\hline 1 & 1.443816700 & -7.768688500 & -2.5750785 \\
\hline 6 & 0.586926500 & -9.144067000 & -3.9982127 \\
\hline 1 & 1.371540000 & -8.971749300 & -4.7348640 \\
\hline 6 & -0.446859700 & -10.051566900 & -4.2677938 \\
\hline 1 & -0.473452000 & -10.587481200 & -5.2165547 \\
\hline 6 & -1.447449100 & -10.273699800 & -3.3055861 \\
\hline 1 & -2.254964700 & -10.978088800 & -3.5058957 \\
\hline 6 & -1.408232400 & -9.598959400 & -2.0780754 \\
\hline 1 & -2.182226000 & -9.804609500 & -1.3397587 \\
\hline 6 & -0.644082000 & -9.094996100 & 1.1061923 \\
\hline 6 & -0.105971600 & -10.387083900 & 0.9208643 \\
\hline 1 & 0.413564100 & -10.641043600 & -0.0011310 \\
\hline 6 & -0.246955400 & -11.363547200 & 1.9196756 \\
\hline 1 & 0.165535900 & -12.359490500 & 1.7577934 \\
\hline 6 & -0.918254100 & -11.063038300 & 3.1149662 \\
\hline 1 & -1.030974100 & -11.824358100 & 3.8 \\
\hline 6 & -1.443312600 & -9.775629200 & 3.3125799 \\
\hline 1 & -1.963287200 & -9.532092000 & 4.2389987 \\
\hline 6 & -1.304091500 & -8.795143600 & 2.3187086 \\
\hline 1 & -1.710564900 & -7.802207800 & 2.5037950 \\
\hline 6 & -0.122032800 & -3.172663800 & -1.6287984 \\
\hline 6 & 0.104922500 & -1.838434200 & -1.2207772 \\
\hline 1 & -0.048977000 & -1.543691100 & -0.1842281 \\
\hline 6 & 0.513774800 & -0.871400600 & -2.1522263 \\
\hline 1 & 0.675 & 21800 & -1.82 \\
\hline 6 & 0.707808800 & -1.221050000 & -3.4976853 \\
\hline 1 & 1.021758400 & -0.467008200 & -4.2191169 \\
\hline 6 & 0.499300600 & -2.547503400 & -3.9084642 \\
\hline 1 & 0.651915300 & -2.828937400 & -4.9503868 \\
\hline 6 & 0.094826300 & -3.520476900 & -2.9821387 \\
\hline 1 & -0.046167900 & -4.543815600 & -3.3262854 \\
\hline 6 & -1.497770500 & -3.626595600 & 0.9594436 \\
\hline 6 & -1.196682300 & -3.882289700 & 2.3142945 \\
\hline 1 & -0.37 & 3900 & 2.57 \\
\hline 6 & -1.951710900 & -3.287875700 & 3.3398582 \\
\hline 1 & -1.702580500 & -3.490787500 & 4.3816169 \\
\hline 6 & -3.016188400 & -2.431398500 & 3.0228167 \\
\hline 1 & -3.598219300 & -1.964370600 & 3.8169623 \\
\hline 6 & -3.328699700 & -2.174492000 & 1.6765318 \\
\hline 1 & -4.155156700 & -1.510042500 & 1.4246295 \\
\hline 6 & -2.577688100 & -2.765747500 & 0.6507241 \\
\hline 1 & -2.829468100 & -2.543854900 & -0.3858383 \\
\hline 8 & 3.161788000 & -7.220430200 & -1.3196730 \\
\hline 6 & 2.964138800 & -7.093026800 & -0.1288147 \\
\hline 1 & 1.404581800 & -7.164265400 & 1.6200400 \\
\hline 6 & 4.026524100 & -7.326767400 & 0.9530768 \\
\hline 1 & 4.954570100 & -7.707056500 & 0.5039273 \\
\hline 1 & 3.630208000 & -8.069686200 & 1.6584260 \\
\hline 6 & 4.220915400 & -5.957749400 & \\
\hline
\end{tabular}




\begin{tabular}{|c|c|c|c|}
\hline 1 & 4.847068900 & -5.319082300 & 1.013192100 \\
\hline 1 & 4.755791400 & -6.096010200 & 2.600965400 \\
\hline 6 & 2.886688100 & -5.258363100 & 1.915399600 \\
\hline 1 & 2.460284900 & -5.388206000 & 2.910915100 \\
\hline 6 & 2.399792200 & -4.229434600 & 1.114398300 \\
\hline 1 & 2.957983500 & -3.894231500 & 0.238250300 \\
\hline 1 & 1.665756100 & -3.528100900 & 1.502751100 \\
\hline \multicolumn{4}{|c|}{67} \\
\hline & $a+7.2$ & -6794.118770 & \\
\hline 45 & -1.233548159 & -3.709297661 & -1.057408824 \\
\hline 15 & -3.200796875 & -4.994109404 & -1.397172032 \\
\hline 6 & -4.626217931 & -3.782474582 & -1.648864585 \\
\hline 1 & -5.426610950 & -4.253125245 & -2.230747879 \\
\hline 1 & -5.041790697 & -3.585551603 & -0.652723613 \\
\hline 6 & -4.170959666 & -2.467552248 & -2.317632987 \\
\hline 1 & -3.827802139 & -2.656595934 & -3.340984702 \\
\hline 1 & -5.005105556 & -1.755350440 & -2.364636792 \\
\hline 15 & -2.728415096 & -1.732179820 & -1.363751264 \\
\hline 6 & -3.232050700 & -6.215268191 & -2.789045141 \\
\hline 6 & -4.390004516 & -6.444156710 & -3.563775930 \\
\hline 1 & -5.310197718 & -5.895381385 & -3.378895225 \\
\hline 6 & -4.383113346 & -7.404776450 & -4.587866336 \\
\hline 1 & -5.285604975 & -7.566050956 & -5.177349732 \\
\hline 6 & -3.226595229 & -8.1556 & -4.846 \\
\hline 1 & -3.224036367 & -8.8989 & -5.64 \\
\hline 6 & -2.075113367 & -7.9467 & -4.07 \\
\hline 1 & -1.175405040 & -8.532088994 & -4.258990079 \\
\hline 6 & -2.075721931 & -6.982428119 & -3.052952844 \\
\hline 1 & -1.177383359 & -6.833744662 & -2.457243290 \\
\hline 6 & -3.676335304 & -5.957483307 & 0.117380295 \\
\hline 6 & -3.908935123 & -5.267297748 & 1.329861066 \\
\hline 1 & -3.848182807 & -4.181001107 & 1.372454535 \\
\hline 6 & -4.233826447 & -5.970053884 & 2.498989883 \\
\hline 1 & $-4.41 \xi-\gamma$ & -5.42 & 3.42 \\
\hline 6 & -4.322501568 & -7.371675290 & 27030 \\
\hline 1 & -4.576392052 & -7.917081499 & 3.386861261 \\
\hline 6 & -4.089149454 & -8.064211580 & 1.281220100 \\
\hline 1 & -4.162808991 & -9.151260136 & 1.254448476 \\
\hline 6 & -3.765871304 & -7.366000802 & 0.106559605 \\
\hline 1 & -3.596666994 & -7.921916803 & -0.812496871 \\
\hline 6 & -2.162401359 & -0.254918829 & -2.331377411 \\
\hline 6 & -2.127507086 & -0.267019113 & -3.744365199 \\
\hline 1 & -2.476152978 & -1.130754428 & -4.304950379 \\
\hline 6 & -1.634415782 & 3533 & -4.45 \\
\hline 1 & -1.625907477 & 0.820982444 & -5.540554446 \\
\hline 6 & -1.157690440 & 1.968435408 & -3.762945128 \\
\hline 1 & -0.778557084 & 2.827877496 & -4.315338967 \\
\hline 6 & -1.176208969 & 1.983776790 & -2.359200891 \\
\hline 1 & -0.812908067 & 2.855579621 & -1.815049544 \\
\hline 6 & -1.673589379 & 0.882156442 & -1.646442536 \\
\hline 1 & -1.696755205 & 0.922840455 & -0.558523040 \\
\hline 6 & -3.526834152 & -1.004245591 & 0.148097847 \\
\hline 6 & -3.066129637 & -1.350730414 & 1.435655423 \\
\hline 1 & -2.229006438 & -2.037292437 & 1.550403326 \\
\hline 6 & -3.671284436 & -0.812223434 & 2.584457963 \\
\hline
\end{tabular}




\begin{tabular}{|c|c|c|c|}
\hline 1 & -3.298538210 & -1.085931285 & 3.571585071 \\
\hline 6 & -4.747544128 & 0.078196420 & 2.458380519 \\
\hline 1 & -5.218758356 & 0.496328231 & 3.347810664 \\
\hline 6 & -5.212395977 & 0.435267619 & 1.180687093 \\
\hline 1 & -6.042564435 & 1.133799662 & 1.076160503 \\
\hline 6 & -4.605901486 & -0.096648665 & 0.034119238 \\
\hline 1 & -4.968042328 & 0.211619631 & -0.945784934 \\
\hline 8 & -1.344088838 & -3.648228977 & -3.922136626 \\
\hline 6 & -0.606409767 & -3.793043812 & -2.972028096 \\
\hline 1 & -0.603481677 & -5.167445620 & -0.866703543 \\
\hline 6 & 0.911930715 & -4.060799243 & -3.102325287 \\
\hline 1 & 1.216580849 & -3.806610000 & -4.126749380 \\
\hline 1 & 1.034414185 & -5.145190096 & -2.969299623 \\
\hline 6 & 1.719240952 & -3.287383882 & -2.038656419 \\
\hline 1 & 1.798291014 & -2.234589050 & -2.343005761 \\
\hline 1 & 2.741134014 & -3.688278602 & -1.988911518 \\
\hline 6 & 1.061668315 & -3.366875090 & -0.666368401 \\
\hline 1 & 1.359546891 & -4.185414085 & -0.012679347 \\
\hline 6 & 0.348392216 & -2.298236840 & -0.139040078 \\
\hline 1 & 0.330765630 & -1.335695683 & -0.647170164 \\
\hline 1 & 0.083611961 & -2.287890304 & 0.920002164 \\
\hline \multicolumn{4}{|c|}{ 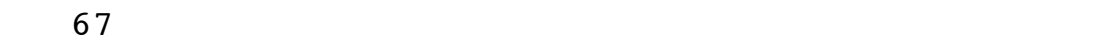 } \\
\hline \multicolumn{4}{|c|}{ T.E. $=-679$} \\
\hline 45 & 0.072914857 & -0.204215125 & -2.164134753 \\
\hline 15 & -1.810504859 & -1.6395 & -2.354 \\
\hline 6 & -3.318911728 & -0.591455684 & -1.968108559 \\
\hline 1 & -4.236471535 & -1.123574880 & -2.251362275 \\
\hline 1 & -3.345787486 & -0.432754008 & -0.882472361 \\
\hline 6 & -3.220829073 & 0.759926724 & -2.710981317 \\
\hline 1 & -3.231814358 & 0.596673045 & -3.795339087 \\
\hline 1 & -4.079002065 & 1.397426301 & -2.463915366 \\
\hline 15 & -1.623261542 & 1.659155845 & -2.245923925 \\
\hline 6 & -2.073052221 & -2.174543509 & -4.111746397 \\
\hline 6 & -1.076590303 & -1.949452909 & -5.084 \\
\hline 1 & -0.135148838 & -1.478629733 & -4.801754425 \\
\hline 6 & -1.273041502 & -2.352862503 & -6.416101463 \\
\hline 1 & -0.491194793 & -2.178781068 & -7.155591013 \\
\hline 6 & -2.467756744 & -2.986459807 & -6.788165148 \\
\hline 1 & -2.620526659 & -3.303351064 & -7.819763018 \\
\hline 6 & -3.464036156 & -3.223082886 & -5.824784504 \\
\hline 1 & -4.388993744 & -3.726140933 & -6.106231028 \\
\hline 6 & -3.26 & -2.82 & -4.495128124 \\
\hline 1 & -4.043812433 & -3.038288263 & -3.759701786 \\
\hline 6 & -1.950461398 & -3.195138626 & -1.372344218 \\
\hline 6 & -1.242183524 & -4.334334308 & -1.816982781 \\
\hline 1 & -0.656820293 & -4.296940688 & -2.734821656 \\
\hline 6 & -1.293327136 & -5.529283469 & -1.086504382 \\
\hline 1 & -0.745705250 & -6.401775632 & -1.442530519 \\
\hline 6 & -2.048782023 & -5.602744828 & 0.095873512 \\
\hline 1 & -2.087936005 & -6.532618389 & 0.662944412 \\
\hline 6 & -2.751480239 & -4.474602084 & 0.544649031 \\
\hline 1 & -3.337892709 & -4.522272571 & 1.462188789 \\
\hline 6 & -2.702045647 & -3.273370394 & -0.181026485 \\
\hline 1 & -3.245208156 & -2.410991584 & 0.197933821 \\
\hline 6 & -1.272025584 & 2.827914755 & -3.644511727 \\
\hline
\end{tabular}




\begin{tabular}{|c|c|c|c|}
\hline 6 & -1.424753144 & 2.417897252 & -4.989289799 \\
\hline 1 & -1.833988760 & 1.439199833 & -5.233523724 \\
\hline 6 & -1.055382092 & 3.270707939 & -6.041728401 \\
\hline 1 & -1.194154902 & 2.942534281 & -7.071908794 \\
\hline 6 & -0.516338374 & 4.538097097 & -5.770916122 \\
\hline 1 & -0.232902221 & 5.199160307 & -6.589598977 \\
\hline 6 & -0.344673816 & 4.948518274 & -4.439471389 \\
\hline 1 & 0.072500882 & 5.930997783 & -4.218342034 \\
\hline 6 & -0.714391935 & 4.101265741 & -3.383228180 \\
\hline 1 & -0.581551634 & 4.444740296 & -2.358681798 \\
\hline 6 & -2.171510608 & 2.739150157 & -0.836408276 \\
\hline 6 & -1.710562228 & 2.506290315 & 0.475130210 \\
\hline 1 & -1.003261267 & 1.707108029 & 0.675157884 \\
\hline 6 & -2.175886930 & 3.290765575 & 1.544884103 \\
\hline 1 & -1.811687992 & 3.096116052 & 2.553613520 \\
\hline 6 & -3.103736467 & 4.316504914 & 1.315338212 \\
\hline 1 & -3.461566972 & 4.926910135 & 2.144242889 \\
\hline 6 & -3.574250153 & 4.552991238 & 0.011905657 \\
\hline 1 & -4.297576282 & 5.347136171 & -0.173661123 \\
\hline 6 & -3.116384292 & 3.770349813 & -1.056781622 \\
\hline 1 & -3.488182844 & 3.974395399 & -2.060085074 \\
\hline 8 & -0.385323232 & -0.955164546 & 0.556028027 \\
\hline 6 & 0.405940284 & -0.436866239 & -0.198141540 \\
\hline 1 & 0.804190328 & -1.604110308 & -2.381295609 \\
\hline 6 & 1.780634607 & 0.126381013 & 0.244258799 \\
\hline 1 & 2.513476830 & -0.662183757 & 0.019529124 \\
\hline 1 & 1.758545999 & 44809753 & 1.335515181 \\
\hline 6 & 2.129110251 & 1385 & 85723662 \\
\hline 1 & 3.195786204 & 1.676233268 & -0.337390994 \\
\hline 1 & 1.563092925 & 2.269968239 & -0.042597195 \\
\hline 6 & 1.815733309 & 1.346711365 & -1.973281982 \\
\hline 1 & 1.502755146 & 2.262170180 & -2.473828442 \\
\hline 6 & 2.218710955 & 0.266829480 & -2.754090632 \\
\hline 1 & 2.875984000 & -0.508521955 & -2.365857937 \\
\hline 1 & 2.179437761 & 0.336391604 & -3.842961699 \\
\hline \multicolumn{4}{|c|}{ 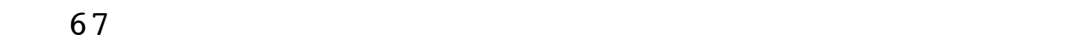 } \\
\hline \multicolumn{4}{|c|}{$2 \mathrm{~b} "+6.3$ T.E. $=-6794.1200760761$} \\
\hline 45 & -0.406952591 & 0.099685806 & -0.102744336 \\
\hline 15 & -2.103318063 & 1.734637241 & -0.195478328 \\
\hline 6 & -1.451124990 & 3.243680064 & 0.703913465 \\
\hline 1 & -2.092941474 & 4.116778714 & 0.523903727 \\
\hline 1 & -1.555153258 & 2.985451705 & 1.765891952 \\
\hline 6 & 0.015893121 & 3.566405442 & 0.342115715 \\
\hline 1 & 0.073644153 & 4.029965945 & -0.649605980 \\
\hline 1 & 0.442398549 & 4.281832706 & 1.057212416 \\
\hline 15 & 1.137550178 & 2.037014113 & 0.232358179 \\
\hline 6 & -2.384490383 & 2.206685397 & -1.967325750 \\
\hline 6 & -2.269394828 & 3.528765067 & -2.448878945 \\
\hline 1 & -2.003967710 & 4.347564198 & -1.783333107 \\
\hline 6 & -2.511371704 & 3.816138594 & -3.802210902 \\
\hline 1 & -2.420207820 & 4.842373151 & -4.157934559 \\
\hline 6 & -2.873792088 & 2.791485585 & -4.689738062 \\
\hline 1 & -3.064182958 & 3.018551758 & -5.738458422 \\
\hline 6 & -2.998095123 & 1.473807875 & -4.219161137 \\
\hline 1 & -3.290490280 & 0.673864276 & -4.899378889 \\
\hline
\end{tabular}




\begin{tabular}{|c|c|c|c|c|}
\hline 6 & & -2.752795606 & 1.181081480 & -2.870278434 \\
\hline 1 & & -2.863183928 & 0.155971118 & -2.518486268 \\
\hline 6 & & -3.798896392 & 1.460107937 & 0.490558376 \\
\hline 6 & & -4.178528912 & 0.215204285 & 1.029349607 \\
\hline 1 & & -3.468532450 & -0.607486572 & 1.057358969 \\
\hline 6 & & -5.476056874 & 0.027006177 & 1.531440278 \\
\hline 1 & & -5.757197156 & -0.940847774 & 1.946425715 \\
\hline 6 & & -6.404084184 & 1.078210039 & 1.503115774 \\
\hline 1 & & -7.410370071 & 0.930481823 & 1.894764167 \\
\hline 6 & & -6.035064421 & 2.321284141 & 0.962449191 \\
\hline 1 & & -6.753045241 & 3.140632553 & 0.929144557 \\
\hline 6 & & -4.742250400 & 2.513032557 & 0.455415593 \\
\hline 1 & & -4.485656328 & 3.478138605 & 0.020889677 \\
\hline 6 & & 2.260056841 & 2.467321345 & -1.179121338 \\
\hline 6 & & 3.166501992 & 3.549826034 & -1.095182747 \\
\hline 1 & & 3.268281090 & 4.113718150 & -0.168956968 \\
\hline 6 & & 3.952347450 & 3.901652145 & -2.202118917 \\
\hline 1 & & 4.648915456 & 4.736531979 & -2.124494181 \\
\hline 6 & & 3.844798574 & 3.181948475 & -3.404730824 \\
\hline 1 & & 4.458729290 & 3.457562538 & -4.262182565 \\
\hline 6 & & 2.946138808 & 2.108916918 & -3.498707320 \\
\hline 1 & & 2.857604747 & 1.547450743 & -4.428711233 \\
\hline 6 & & 2.157254798 & 1.753925641 & -2.391959644 \\
\hline 1 & & 1.457243819 & 0.923815069 & -2.482761707 \\
\hline 6 & & 2.213658738 & 2.099708749 & 1.740934765 \\
\hline 6 & & 3.549148722 & 1.638281297 & 1.681560321 \\
\hline 1 & & 3.978375272 & 1.312334741 & 0.735206628 \\
\hline 6 & & 4.348414978 & 1.621380400 & 2.835241786 \\
\hline 1 & & 5.380403671 & 1.276606585 & 2.768147323 \\
\hline 6 & & 3.826526885 & 2.053975243 & 4.065150859 \\
\hline 1 & & 4.449850123 & 2.043789160 & 4.959182062 \\
\hline 6 & & 2.499006348 & 2.504247802 & 4.135892087 \\
\hline 1 & & 2.084286972 & 2.842336344 & 5.085619975 \\
\hline 6 & & 1.695032004 & 2.524418547 & 2.984867608 \\
\hline 1 & & 0.667104976 & 2.870301327 & 3.073221190 \\
\hline 8 & & -1.142492467 & 0.303163815 & 2.652125911 \\
\hline 6 & & -0.528606736 & -0.363303121 & 1.849216932 \\
\hline 1 & & -1.663378260 & -0.844307736 & -0.396853612 \\
\hline 6 & & 0.257789447 & -1.646075902 & 2.211803116 \\
\hline 1 & & -0.402861643 & -2.487718477 & 1.956443205 \\
\hline 1 & & 0.398343514 & -1.655832366 & 3.300168455 \\
\hline 6 & & 1.594428320 & -1.731369042 & 1.444665871 \\
\hline 1 & & 2.016548603 & -2.745046652 & 1.542397812 \\
\hline 1 & & 2.316680638 & -1.044417474 & 1.900393680 \\
\hline 6 & & 1.413620874 & -1.378790551 & -0.026256251 \\
\hline 1 & & 2.259455527 & -0.908809148 & -0.527573066 \\
\hline 6 & & 0.391205169 & -1.913500139 & -0.806218932 \\
\hline 1 & & -0.258951247 & -2.701861124 & -0.432491794 \\
\hline 1 & & 0.424019478 & -1.824840464 & -1.893949341 \\
\hline & 67 & & & \\
\hline $2 \mathrm{~b}$ & +8.3 & \multicolumn{3}{|c|}{ T.E. $=-6794.1168283864$} \\
\hline 45 & & -0.409254640 & 0.118485751 & -0.148481865 \\
\hline 15 & & -2.105827893 & 1.734665248 & -0.202507716 \\
\hline 6 & & -1.443439906 & 3.254449878 & 0.671204420 \\
\hline 1 & & -2.091943261 & 4.123713973 & 0.496462834 \\
\hline
\end{tabular}




\begin{tabular}{|c|c|c|c|}
\hline 1 & -1.529179356 & 3.004588430 & 1.736828126 \\
\hline 6 & 0.017041813 & 3.583817288 & 0.284195359 \\
\hline 1 & 0.057556208 & 4.040198700 & -0.711759039 \\
\hline 1 & 0.447210552 & 4.311132766 & 0.985285844 \\
\hline 15 & 1.149856740 & 2.065197539 & 0.162227427 \\
\hline 6 & -2.400135224 & 2.180726175 & -1.978136752 \\
\hline 6 & -2.287120808 & 3.495104658 & -2.481588814 \\
\hline 1 & -2.012091622 & 4.323372636 & -1.831866942 \\
\hline 6 & -2.545040866 & 3.761730540 & -3.836052146 \\
\hline 1 & -2.456544561 & 4.782214579 & -4.208579219 \\
\hline 6 & -2.919764678 & 2.724086817 & -4.703472768 \\
\hline 1 & -3.123156132 & 2.935661176 & -5.753004221 \\
\hline 6 & -3.040113954 & 1.413823458 & -4.211640690 \\
\hline 1 & -3.341987553 & 0.604081999 & -4.875956773 \\
\hline 6 & -2.780196848 & 1.141578175 & -2.861201701 \\
\hline 1 & -2.888941916 & 0.122615491 & -2.491752039 \\
\hline 6 & -3.792980598 & 1.460327256 & 0.500553698 \\
\hline 6 & -4.178843426 & 0.201447092 & 1.001520346 \\
\hline 1 & -3.473824048 & -0.626072970 & 0.999933213 \\
\hline 6 & -5.474699260 & 0.008424971 & 1.505855872 \\
\hline 1 & -5.761097933 & -0.969539084 & 1.892592612 \\
\hline 6 & -6.394288993 & 1.067475016 & 1.516444177 \\
\hline 1 & -7.399478959 & 0.915463112 & 1.909327895 \\
\hline 6 & -6.018290849 & 2.324499646 & 1.014181684 \\
\hline 1 & -6.729553366 & 3.150324271 & 1.011871376 \\
\hline 6 & -4.727113439 & 2.521892478 & 0.505039380 \\
\hline 1 & -4.465160508 & 3.498819272 & 0.101250073 \\
\hline 6 & 2.308432481 & 2.553634498 & -1.202112351 \\
\hline 6 & 3.282588760 & 3.562568039 & -1.021573582 \\
\hline 1 & 3.419412037 & 4.029464207 & -0.047224641 \\
\hline 6 & 4.090252001 & 3.966463237 & -2.094867347 \\
\hline 1 & 4.838644650 & 4.744333714 & -1.942135801 \\
\hline 6 & 3.936827846 & 3.373638966 & -3.359677638 \\
\hline 1 & 4.567288869 & 3.689009753 & -4.190975439 \\
\hline 6 & 2.970025854 & 2.374583472 & -3.549649225 \\
\hline 1 & 2.843616510 & 1.911761196 & -4.528468223 \\
\hline 6 & 2.160074223 & 1.967051783 & -2.476963546 \\
\hline 1 & 1.405422185 & 1.199439946 & -2.642944807 \\
\hline 6 & 2.193862156 & 2.084535339 & 1.695410436 \\
\hline 6 & 3.488543793 & 1.513960368 & 1.669550053 \\
\hline 1 & 3.905230324 & 1.135741085 & 0.736622391 \\
\hline 6 & 4.268102729 & 1.459686839 & 2.835233948 \\
\hline 1 & 5.269221792 & 1.030440764 & 2.792434315 \\
\hline 6 & 3.766529342 & 1.964044044 & 4.046771215 \\
\hline 1 & 4.374968830 & 1.926441612 & 4.950188019 \\
\hline 6 & 2.478902560 & 2.520355220 & 4.085914515 \\
\hline 1 & 2.079894807 & 2.914042099 & 5.020926487 \\
\hline 6 & 1.695289345 & 2.578882429 & 2.921514046 \\
\hline 1 & 0.699086831 & 3.011673196 & 2.986437247 \\
\hline 8 & -1.195277635 & 0.317809648 & 2.583879108 \\
\hline 6 & -0.479752907 & -0.296638255 & 1.822884787 \\
\hline 1 & -1.616452352 & -0.906577462 & -0.354466446 \\
\hline 6 & 0.523207794 & -1.370686509 & 2.312508197 \\
\hline 1 & 0.038936583 & -1.909462486 & 3.138778986 \\
\hline 1 & 1.352415063 & -0.791359036 & 2.745313758 \\
\hline
\end{tabular}




\begin{tabular}{|c|c|c|c|}
\hline 6 & 1.026692510 & -2.316080702 & 1.208214640 \\
\hline 1 & 0.261642504 & -3.072671335 & 0.988635211 \\
\hline 1 & 1.921976932 & -2.849345326 & 1.560835803 \\
\hline 6 & 1.346248569 & -1.533320447 & -0.051160243 \\
\hline 1 & 2.268722098 & -0.953496599 & -0.061034327 \\
\hline 6 & 0.621963510 & -1.686820190 & -1.214829621 \\
\hline 1 & -0.122373696 & -2.474548665 & -1.312495851 \\
\hline 1 & 0.953882078 & -1.222727629 & -2.145534310 \\
\hline \multicolumn{4}{|c|}{67} \\
\hline \multicolumn{4}{|c|}{ TS2sigma-2b' +16.1 T.E. $=-6794.104504$} \\
\hline 45 & 0.058088986 & -0.085470008 & -2.078027465 \\
\hline 15 & -1.779113471 & -1.598882637 & -2.413888138 \\
\hline 6 & -3.346156519 & -0.563668656 & -2.285506466 \\
\hline 1 & -4.201273612 & -1.120271228 & -2.690317300 \\
\hline 1 & -3.547155710 & -0.368765089 & -1.224468935 \\
\hline 6 & -3.148555329 & 0.765772091 & -3.045945605 \\
\hline 1 & -3.017532538 & 0.575870581 & -4.118443852 \\
\hline 1 & -4.022403847 & 1.419446184 & -2.927471968 \\
\hline 15 & -1.612461427 & 1.641489201 & -2.395393515 \\
\hline 6 & -1.853773175 & -2.255616819 & -4.147936711 \\
\hline 6 & -0.833939445 & -1.955216715 & -5.074481200 \\
\hline 1 & 0.021700637 & -1.357139596 & -4.760898481 \\
\hline 6 & -0.908690387 & -2.432186057 & -6.394465707 \\
\hline 1 & -0.111889621 & -2.197698539 & -7.100529651 \\
\hline 6 & -2.000303722 & -3.214903365 & -6.799261840 \\
\hline 1 & -2.058166808 & -3.587441925 & -7.821838611 \\
\hline 6 & -3.018156057 & -3.524958704 & -5.880224118 \\
\hline 1 & -3.863839832 & -4.140941341 & -6.186420808 \\
\hline 6 & -2.947419115 & -3.051390796 & -4.562756423 \\
\hline 1 & -3.735441956 & -3.319417491 & -3.859914347 \\
\hline 6 & -2.014684220 & -3.072482476 & -1.332018883 \\
\hline 6 & -1.362443879 & -4.280274956 & -1.666648071 \\
\hline 1 & -0.792253181 & -4.360838650 & -2.590953331 \\
\hline 6 & -1.445776786 & -5.389344347 & -0.813 \\
\hline 1 & -0.938147188 & -6.315106560 & -1.083745695 \\
\hline 6 & -2.176461246 & -5.307639445 & 0.383102528 \\
\hline 1 & -2.236932466 & -6.170203995 & 1.046584684 \\
\hline 6 & -2.824259607 & -4.110242085 & 0.723515753 \\
\hline 1 & -3.392330268 & -4.039320381 & 1.651025483 \\
\hline 6 & -2.739589494 & -2.994856276 & -0.123341341 \\
\hline 1 & -3.236280770 & -2.073756449 & 0.175278149 \\
\hline 6 & -1.228690270 & 2.993222141 & -3.600596545 \\
\hline 6 & -1.281610967 & 2.747730002 & -4.992644018 \\
\hline 1 & -1.616509379 & 1.786140609 & -5.378532679 \\
\hline 6 & -0.904695477 & 3.744283202 & -5.906004506 \\
\hline 1 & -0.963676900 & 3.542381112 & -6.975622564 \\
\hline 6 & -0.454961929 & 4.992863536 & -5.446735815 \\
\hline 1 & -0.163240864 & 5.765287391 & -6.157957541 \\
\hline 6 & -0.382761984 & 5.240338939 & -4.067188380 \\
\hline 1 & -0.034159968 & 6.206102047 & -3.700637591 \\
\hline 6 & -0.762932537 & 4.249460301 & -3.148430037 \\
\hline 1 & -0.711460918 & 4.467451252 & -2.083090143 \\
\hline 6 & -2.223309629 & 2.477707785 & -0.859806326 \\
\hline 6 & -1.737909165 & 2.086457029 & 0.405471078 \\
\hline 1 & -0.977766395 & 1.309895378 & 0.479878482 \\
\hline
\end{tabular}




\begin{tabular}{|c|c|c|c|}
\hline 6 & -2.236238176 & 2.687562266 & 1.573983627 \\
\hline 1 & -1.853396957 & 2.376523459 & 2.546003867 \\
\hline 6 & -3.220803765 & 3.683446986 & 1.489993682 \\
\hline 1 & -3.604133990 & 4.152013856 & 2.396239929 \\
\hline 6 & -3.712818219 & 4.076405720 & 0.233079415 \\
\hline 1 & -4.477282716 & 4.849940931 & 0.159845300 \\
\hline 6 & -3.221092905 & 3.478053131 & -0.935499007 \\
\hline 1 & -3.608436017 & 3.803190700 & -1.900555737 \\
\hline 8 & 0.593658634 & -1.923648352 & 0.152 \\
\hline 6 & 1.161859879 & -1.145904485 & -0.587718008 \\
\hline 1 & 1.034066928 & -1.426622199 & -2.029094438 \\
\hline 6 & 2.542875199 & -0.527265212 & -0.313604250 \\
\hline 1 & 3.232640704 & -0.912516162 & -1.079065156 \\
\hline 1 & 2.889065036 & -0.886094924 & 0.663831473 \\
\hline 6 & 2.464219392 & 1.012488474 & -0.364988276 \\
\hline 1 & 3.479688849 & 1.441931035 & -0.349763024 \\
\hline 1 & 1.961139127 & 1.376786266 & 0.541385045 \\
\hline 6 & 1.709076579 & 1.512534957 & -1.590040953 \\
\hline 1 & 1.219219546 & 2.479298270 & -1.473407487 \\
\hline 6 & 1.868465626 & 1.010376534 & -2.878445669 \\
\hline 1 & 2.613432152 & 0.247877862 & -3.104198244 \\
\hline 1 & 1.510329503 & 1.582070773 & -3.734112178 \\
\hline \multicolumn{4}{|r|}{ 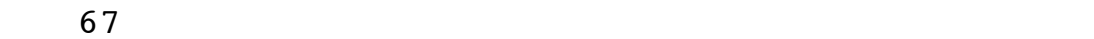 } \\
\hline & +9.3 & -6794.115269 & \\
\hline 45 & 0.058427713 & -0.196792860 & -2.156222487 \\
\hline 15 & -1.828236030 & -1.647204892 & -2.326254858 \\
\hline 6 & -3.325401251 & -0.606638542 & -1.892902191 \\
\hline 1 & -4.253733032 & -1.142431629 & -2.129514011 \\
\hline 1 & -3.307143477 & -0.459264138 & -0.804950260 \\
\hline 6 & -3.249709768 & 0.740508056 & -2.645638800 \\
\hline 1 & -3.248973516 & 0.576006538 & -3.730705248 \\
\hline 1 & -4.127064405 & 1.361134525 & -2.430905516 \\
\hline 15 & -1.657054258 & 1.672486196 & -2.257234334 \\
\hline 6 & -2.072852905 & -2.101049299 & -4.110182455 \\
\hline 6 & -0.950435846 & -2.266436973 & -4.952438119 \\
\hline 1 & 0.054460519 & -2.136800185 & -4.552602444 \\
\hline 6 & -1.1140 & -2 . & -6 . \\
\hline 1 & -0 & -2.75 & -6.934835803 \\
\hline 6 & -2.400236550 & -2.817643562 & -6.827787891 \\
\hline 1 & -2.528371545 & -3.094592958 & -7.874032351 \\
\hline 6 & -3.523088879 & -2.662202058 & -5.998484088 \\
\hline 1 & -4.525209549 & -2.821847890 & -6.396384770 \\
\hline 6 & -3.363018965 & -2.310645199 & -4.649983494 \\
\hline 1 & -4.250621668 & -2.219098426 & -4.027117382 \\
\hline 6 & -1.976788766 & -3.237372802 & -1.392884203 \\
\hline 6 & -1.99263 & 472660153 & -2.078468641 \\
\hline 1 & -1.953130861 & -4.504042082 & -3.164075541 \\
\hline 6 & -2.064981177 & -5.678701510 & -1.364200148 \\
\hline 1 & -2.082062978 & -6.622668005 & -1.908852978 \\
\hline 6 & -2.114324189 & -5.669822275 & 0.037636516 \\
\hline 1 & -2.164770671 & -6.607792115 & 0.590285321 \\
\hline 6 & -2.098076477 & -4.445629380 & 0.725019058 \\
\hline 1 & -2.133588785 & -4.429149040 & 1.813950009 \\
\hline 6 & -2.029086878 & -3.234733625 & 0.019818353 \\
\hline 1 & -1.999067072 & -2.301638089 & 0.574937179 \\
\hline
\end{tabular}




\begin{tabular}{|c|c|c|c|}
\hline 6 & -1.455811155 & 2.816302491 & -3.706243241 \\
\hline 6 & -1.107907004 & 2.248058473 & -4.954300597 \\
\hline 1 & -0.986322537 & 1.169242225 & -5.057272057 \\
\hline 6 & -0.923827345 & 3.060775754 & -6.081845445 \\
\hline 1 & -0.664610038 & 2.606658333 & -7.038386752 \\
\hline 6 & -1.070707608 & 4.454134681 & -5.976793006 \\
\hline 1 & -0.922091405 & 5.086856383 & -6.851540629 \\
\hline 6 & -1.407675662 & 5.026715648 & -4.741176213 \\
\hline 1 & -1.524405644 & 6.106919014 & -4.65321387 \\
\hline 6 & -1.601488950 & 4.215897455 & -3.609834031 \\
\hline 1 & -1.867380740 & 4.678544427 & -2.661628256 \\
\hline 6 & -2.060508137 & 2.752347025 & -0.798503751 \\
\hline 6 & -0.998290484 & 3.352022845 & -0.086712287 \\
\hline 1 & 0.027408131 & 3.184026666 & -0.402611637 \\
\hline 6 & -1.243422760 & 4.176668913 & 1.020515933 \\
\hline 1 & -0.407596149 & 4.627771062 & 1.554735709 \\
\hline 6 & -2.560653949 & 4.413737144 & 1.443723749 \\
\hline 1 & -2.754611239 & 5.048446785 & 2.307975287 \\
\hline 6 & -3.626299787 & 3.830123423 & 0.743806976 \\
\hline 1 & -4.653714738 & 4.012958241 & 1.058063921 \\
\hline 6 & -3.381267676 & 3.007781634 & -0.368059421 \\
\hline 1 & -4.236019261 & 2.584310302 & -0.888312918 \\
\hline 8 & -0.509162864 & -0.407465850 & 0.627701311 \\
\hline 6 & 0.361845411 & -0.163731943 & -0.176158050 \\
\hline 1 & 0.764374009 & -1.622581084 & -2.227322533 \\
\hline 6 & 1.810559084 & 0.229067477 & 0.202308303 \\
\hline 1 & 2.421041638 & -0.669798159 & 0.036729036 \\
\hline 1 & 1.830164603 & 0.450280872 & 1.277416127 \\
\hline 6 & 2.318728849 & 1.4124 & -0.6 \\
\hline 1 & 3.416566837 & 1.476706937 & -0.581259537 \\
\hline 1 & 1.931942848 & 2.351272494 & -0.240018176 \\
\hline 6 & 1.894725036 & 1.268684827 & -2.110892885 \\
\hline 1 & 1.609057197 & 2.174604314 & -2.644755480 \\
\hline 6 & 2.192879643 & 0.122492799 & -2.844210230 \\
\hline 1 & 2.838853304 & -0.655417271 & -2.441601626 \\
\hline 1 & 2.098976838 & 0.128277938 & -3.932167730 \\
\hline \multicolumn{4}{|c|}{ 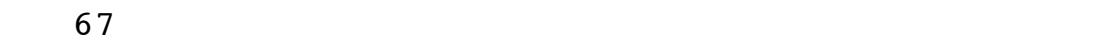 } \\
\hline & $0+10.0$ & -6794.114220 & \\
\hline 45 & -0.410546800 & 0.096759700 & -0.105765300 \\
\hline 15 & -2.106283100 & 1.733114600 & -0.202972500 \\
\hline 6 & -1.452643600 & 3.246332300 & 0.688480300 \\
\hline 1 & -2.090372300 & 4.120295800 & 0.498790300 \\
\hline 1 & -1.564612600 & 2.996059800 & 1.751385100 \\
\hline 6 & 0.018159300 & 3.564587200 & 0.335544500 \\
\hline 1 & 0.084869900 & 4.025774200 & -0.656810600 \\
\hline 1 & 0.439753300 & 4.283293000 & 1.050610300 \\
\hline 15 & 1.14 & 2.036078400 & 0.23648270 \\
\hline 6 & -2.374638300 & 2.190628600 & -1.980019000 \\
\hline 6 & -2.248325800 & 3.507184600 & -2.473717900 \\
\hline 1 & -1.980222200 & 4.330401200 & -1.814719900 \\
\hline 6 & -2.482984300 & 3.783040300 & -3.830677700 \\
\hline 1 & -2.384024900 & 4.805254600 & -4.195743100 \\
\hline 6 & -2.848057500 & 2.752097200 & -4.709970600 \\
\hline 1 & -3.032759400 & 2.970405800 & -5.761554500 \\
\hline 6 & -2.982453400 & 1.439625500 & -4.227553600 \\
\hline
\end{tabular}




\begin{tabular}{|c|c|c|c|}
\hline 1 & -3.277023800 & 0.635062200 & -4.901417200 \\
\hline 6 & -2.745213800 & 1.158462400 & -2.874803400 \\
\hline 1 & -2.863970600 & 0.137636600 & -2.513485000 \\
\hline 6 & -3.805962900 & 1.467002000 & 0.474552500 \\
\hline 6 & -4.200060800 & 0.216200400 & 0.989041800 \\
\hline 1 & -3.496707000 & -0.612541700 & 1.008637600 \\
\hline 6 & -5.502600800 & 0.031786700 & 1.479319200 \\
\hline 1 & -5.795614200 & -0.940384400 & 1.875598600 \\
\hline 6 & -6.420781200 & 1.091966200 & 1.463140500 \\
\hline 1 & -7.431093100 & 0.946805300 & 1.845265300 \\
\hline 6 & -6.036739300 & 2.340997500 & 0.947220000 \\
\hline 1 & -6.746737800 & 3.167602200 & 0.923957400 \\
\hline 6 & -4.739023600 & 2.529212900 & 0.451396700 \\
\hline 1 & -4.470699400 & 3.499332200 & 0.035554000 \\
\hline 6 & 2.301579400 & 2.491263800 & -1.137030300 \\
\hline 6 & 3.246094300 & 3.533146300 & -0.985893500 \\
\hline 1 & 3.357781200 & 4.041859900 & -0.029410800 \\
\hline 6 & 4.056679700 & 3.915153100 & -2.064700000 \\
\hline 1 & 4.782413900 & 4.718145700 & -1.934939500 \\
\hline 6 & 3.936167900 & 3.266372800 & -3.305594500 \\
\hline 1 & 4.568721900 & 3.565447900 & -4.141330900 \\
\hline 6 & 3.000539300 & 2.233402800 & -3.465898000 \\
\hline 1 & 2.900992700 & 1.727967600 & -4.426541100 \\
\hline 6 & 2.186630500 & 1.848278500 & -2.387637900 \\
\hline 1 & 1.456552700 & 1.052353900 & -2.530262700 \\
\hline 6 & 2.181719000 & 2.072331200 & 1.771131100 \\
\hline 6 & 3.492867700 & 1.542628200 & 1.748169700 \\
\hline 1 & 3.924316000 & 1.178855900 & 0.816523100 \\
\hline 6 & 4.268562600 & 1.510246600 & 2.917221500 \\
\hline 1 & 5.283376900 & 1.114155800 & 2.877288500 \\
\hline 6 & 3.746502800 & 1.995598700 & 4.127644100 \\
\hline 1 & 4.352465800 & 1.975368200 & 5.033323100 \\
\hline 6 & 2.441607100 & 2.510788900 & 4.163293000 \\
\hline 1 & 2.026368400 & 2.888868100 & 5.097617500 \\
\hline 6 & 1.661504800 & 2.547025500 & 2.996000300 \\
\hline 1 & 0.651110800 & 2.946038300 & 3.057632900 \\
\hline 8 & -1.2144 & 0.3 & $2.6 c$ \\
\hline 6 & -0.521898200 & -0.320255700 & 1.849211000 \\
\hline 1 & -1.646683000 & -0.879561600 & -0.375717700 \\
\hline 6 & 0.356323200 & -1.487442600 & 2.360852600 \\
\hline 1 & -0.358043400 & -2.224289600 & 2.757072600 \\
\hline 1 & 0.881105300 & -1.074832900 & 3.233192300 \\
\hline 6 & 1.340995800 & -2.133833000 & 1.348666700 \\
\hline 1 & 1.069145100 & -3.188025100 & 1.201271400 \\
\hline 1 & 2.350214100 & -2.129398300 & 1.778099600 \\
\hline 6 & 1.370703900 & -1.444482500 & -0.014314500 \\
\hline 1 & 2.260464100 & -0.871206300 & -0.269244100 \\
\hline 6 & 0.480746100 & -1.793645400 & -1.020497200 \\
\hline 1 & -0.197649900 & -2.636296500 & -0.903173900 \\
\hline 1 & 0.639663500 & -1.457975000 & -2.048307800 \\
\hline \multicolumn{4}{|l|}{67} \\
\hline $2 d$ & \multicolumn{3}{|c|}{ T.E. $=-6794.1017408144$} \\
\hline 45 & 0.129812347 & 0.220700792 & -1.90710467 \\
\hline 15 & -1.518149294 & -1.402701735 & -2.346390465 \\
\hline 6 & -3.178205836 & -0.517477452 & -2.46941796 \\
\hline
\end{tabular}




\begin{tabular}{|c|c|c|c|}
\hline 1 & -3.892046261 & -1.162420336 & -2.997035630 \\
\hline 1 & -3.564579981 & -0.372372674 & -1.453128661 \\
\hline 6 & -3.035987435 & 0.842792706 & -3.186594332 \\
\hline 1 & -2.689241878 & 0.700027805 & -4.217619584 \\
\hline 1 & -4.005795184 & 1.355192236 & -3.232696810 \\
\hline 15 & -1.766863602 & 1.896115682 & -2.277990651 \\
\hline 6 & -1.429659884 & -2.417249045 & -3.891295392 \\
\hline 6 & -0.339406608 & -2.320669700 & -4.778370315 \\
\hline 1 & 0.502377444 & -1.674595576 & -4.542059056 \\
\hline 6 & -0.316947070 & -3.076487313 & -5.961818244 \\
\hline 1 & 0.535174121 & -2.994329846 & -6.636509151 \\
\hline 6 & -1.378276749 & -3.940743948 & -6.268011896 \\
\hline 1 & -1.358815174 & -4.528748888 & -7.185546165 \\
\hline 6 & -2.463756377 & -4.054593555 & -5.382885435 \\
\hline 1 & -3.287573578 & -4.731964440 & -5.607642504 \\
\hline 6 & -2.491301550 & -3.301315848 & -4.200851313 \\
\hline 1 & -3.333676818 & -3.419982529 & -3.520213541 \\
\hline 6 & -1.682271488 & -2.593078498 & -0.942071836 \\
\hline 6 & -1.114673793 & -3.883648229 & -1.036399793 \\
\hline 1 & -0.624796591 & -4.202160626 & -1.953159651 \\
\hline 6 & -1.176290044 & -4.764176726 & 0.053186810 \\
\hline 1 & -0.741954585 & -5.759619281 & -0.038351846 \\
\hline 6 & -1.791094803 & -4.368982789 & 1.252161206 \\
\hline 1 & -1.838790133 & -5.057270346 & 2.095880823 \\
\hline 6 & -2.341620573 & -3.081880354 & 1.361257251 \\
\hline 1 & -2.820032383 & -2.766881033 & 2.288636131 \\
\hline 6 & -2.285177458 & -2.195222781 & 0.275058874 \\
\hline 1 & -2.720232917 & -1.203384291 & 0.389049134 \\
\hline 6 & -1.418116168 & 3.332811750 & -3.394729240 \\
\hline 6 & -1.181103150 & 3.121760432 & -4.773730932 \\
\hline 1 & -1.280424991 & 2.129509709 & -5.211393747 \\
\hline 6 & -0.815932746 & 4.189989941 & -5.607193795 \\
\hline 1 & -0.650325096 & 4.009947736 & -6.669453513 \\
\hline 6 & -0.664762124 & 5.481794136 & -5.077794080 \\
\hline 1 & -0.380601088 & 6.310308492 & -5.726263416 \\
\hline 6 & -0.880666249 & 5.698950116 & -3.707778649 \\
\hline 1 & -0.765507241 & 6.698223981 & -3.287452514 \\
\hline 6 & -1.250826988 & 4.634936193 & -2.869595887 \\
\hline 1 & -1.421389839 & 4.828104775 & -1.811676634 \\
\hline 6 & -2.731783416 & 2.592656709 & -0.853677264 \\
\hline 6 & -2.278174362 & 2.358965506 & 0.462338252 \\
\hline 1 & -1.359987175 & 1.795875772 & 0.631077653 \\
\hline 6 & -2.993813509 & 2.849018453 & 1.567739898 \\
\hline 1 & -2.627415727 & 2.662567486 & 2.577328732 \\
\hline 6 & -4.174790899 & 3.579372625 & 1.369129533 \\
\hline 1 & -4.731560058 & 3.961549687 & 2.224413175 \\
\hline 6 & -4.636886792 & 3.820119776 & 0.063231711 \\
\hline 1 & -5.551897429 & 4.390708259 & -0.095799044 \\
\hline 6 & -3.922753750 & 3.332757685 & -1.040601782 \\
\hline 1 & -4.289781263 & 3.543130518 & -2.044592210 \\
\hline 8 & 1.725921488 & -2.296385906 & -2.043845612 \\
\hline 6 & 1.647559863 & -1.166429585 & -1.592281089 \\
\hline 1 & 0.496340527 & 0.048896598 & -3.386240453 \\
\hline 6 & 2.768520249 & -0.628722394 & -0.650659578 \\
\hline 1 & 3.658800474 & -0.508666108 & -1.286537272 \\
\hline
\end{tabular}




$\begin{array}{rrrr}1 & 3.007878507 & -1.424446735 & 0.067567060 \\ 6 & 2.400453332 & 0.686860041 & 0.064037572 \\ 1 & 3.303761044 & 1.168548872 & 0.476324405 \\ 1 & 1.760077240 & 0.474900693 & 0.934178077 \\ 6 & 1.685982403 & 1.671249824 & -0.846374861 \\ 1 & 1.079608867 & 2.433091484 & -0.350843361 \\ 6 & 1.896209317 & 1.796413290 & -2.204350469 \\ 1 & 2.668888770 & 1.230159597 & -2.720615353 \\ 1 & 1.470963417 & 2.633135573 & -2.754280569\end{array}$

Figure S1. Gibbs Free Energies for the Bosnich Oxidative Addition Pathway from 1 to $2 \mathrm{a}$.

Gibbs Free Energies Bosnich NEB OA Path to 2a

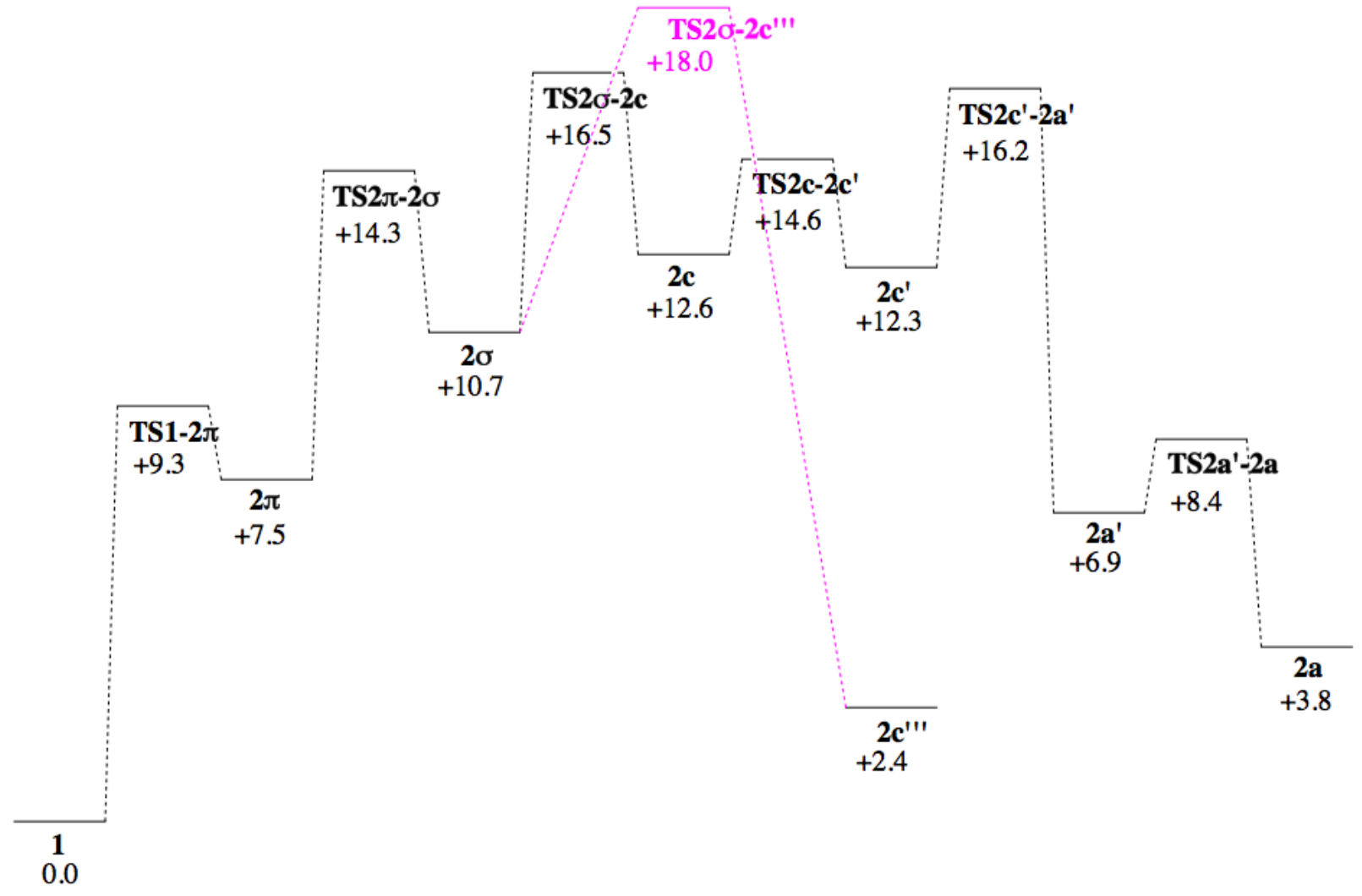

Comparison to OM paper 6-7a $\Delta \mathrm{G}=+18.1 \quad 6-7 \mathrm{~b} \quad \Delta \mathrm{G}=+15.1$ 
Figure S2. Gibbs Free Energies for the Bosnich Oxidative Addition Pathway from 1 to $2 \mathrm{~b}$.

\section{Gibbs Free Energies Bosnich NEB OA Path to $2 b$}

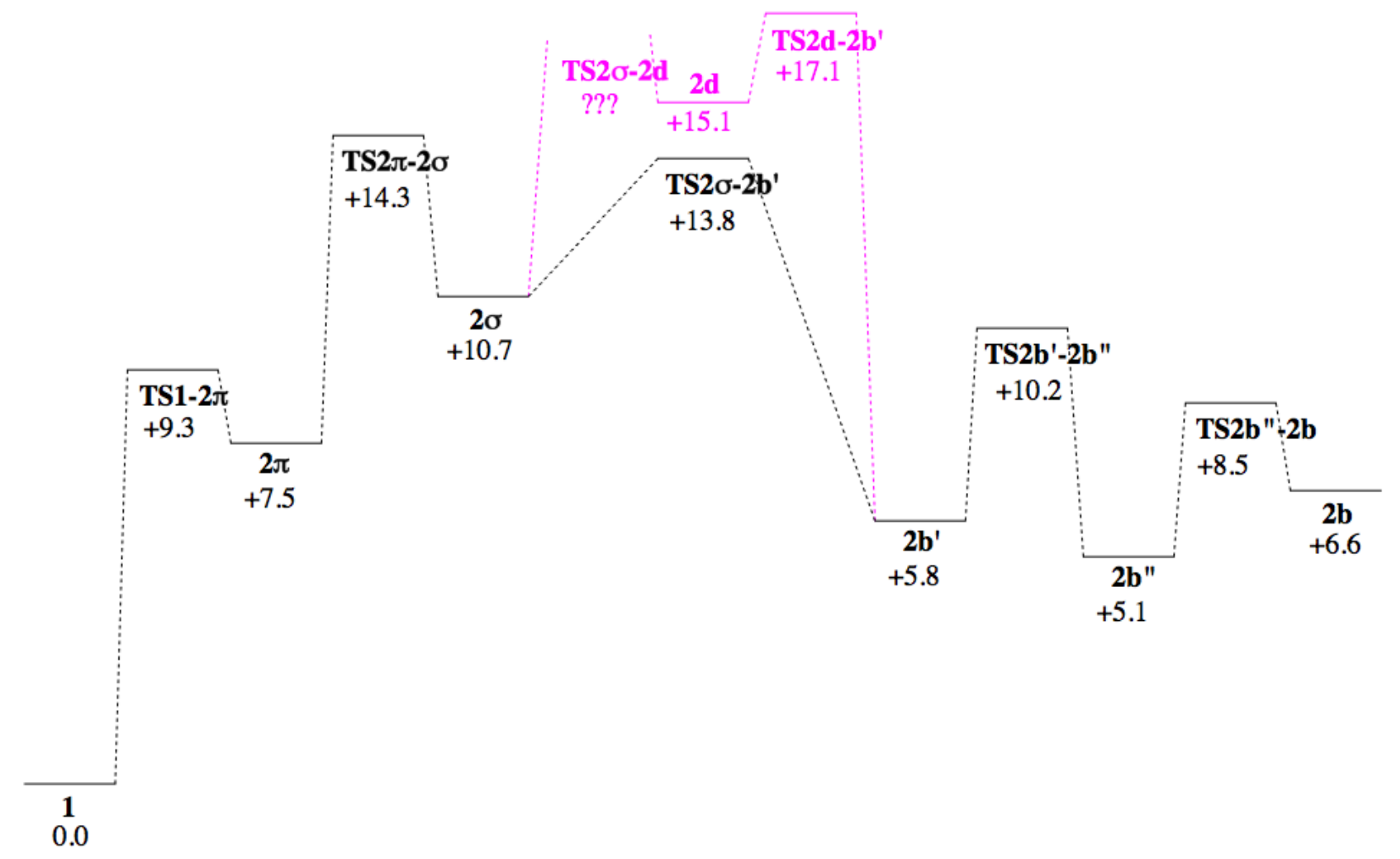

Comparison to OM paper 6-7a $\Delta \mathrm{G}=+18.1 \quad 6-7 \mathrm{~b} \quad \Delta \mathrm{G}=+15.1$ 